TRANSACTIONS OF THE

AMERICAN MATHEMATICAL SOCIETY

Volume 351, Number 9, Pages 3499-3531

S 0002-9947(99)02471-X

Article electronically published on March 29, 1999

\title{
ON MINIMAL PARABOLIC FUNCTIONS AND TIME-HOMOGENEOUS PARABOLIC $h$-TRANSFORMS
}

\author{
KRZYSZTOF BURDZY AND THOMAS S. SALISBURY
}

\begin{abstract}
Does a minimal harmonic function $h$ remain minimal when it is viewed as a parabolic function? The question is answered for a class of long thin semi-infinite tubes $D \subset \mathbb{R}^{d}$ of variable width and minimal harmonic functions $h$ corresponding to the boundary point of $D$ "at infinity." Suppose $f(u)$ is the width of the tube $u$ units away from its endpoint and $f$ is a Lipschitz function. The answer to the question is affirmative if and only if $\int^{\infty} f^{3}(u) d u=\infty$. If the test fails, there exist parabolic $h$-transforms of space-time Brownian motion in $D$ with infinite lifetime which are not time-homogenous.
\end{abstract}

\section{INTRODUCTION AND MAIN RESULTS}

We want to compare the parabolic Martin boundary of a domain in $\mathbb{R}^{d}$ with its Martin boundary, both topologically and probabilistically. In many cases, the two boundaries are related in a very simple way. This provides a complete description of the parabolic Martin boundary in those cases (quite many) when the Martin boundary is known. We plan to present a detailed discussion of this general problem in a separate publication. This paper is devoted to a narrower aspect of the relationship between the two boundaries. We will start with a very informal discussion of a special case which motivated our study. The concepts of the usual and parabolic Martin boundary will be reviewed in a rigorous way later in the introduction. The basic ideas of classical potential theory and Brownian motion may be found in Doob's book ([Db], 1984), to which we will frequently refer.

Consider a strip $D=\left\{\left(x^{1}, x^{2}\right) \in \mathbb{R}^{2}:\left|x^{2}\right|<1\right\}$. Let $X_{t}$ be a Brownian motion starting from $(0,0)$. Then $\dot{X}_{t}=\left(X_{t},-t\right)$ is a space-time Brownian motion starting from $(0,0,0)$. First fix some $s>0$, a point $z \in \partial D$ and a sequence of points $\left\{z_{k}\right\}$ in $D$ converging to $z$ as $k \rightarrow \infty$. Condition $\dot{X}$ to be at $\left(z_{k},-s\right)$ at time $s$ and to not leave $D \times \mathbb{R}$ before time $s$. Then let $k$ go to infinity. The conditioned processes converge in distribution to a process whose first coordinate is a Brownian motion conditioned to exit $D$ through $z$ at time $s$. The lifetime of this process is finite. This conditioned space-time Brownian motion is not time-homogeneous, i.e., its

Received by the editors December 9, 1997 and, in revised form, November 6, 1998.

1991 Mathematics Subject Classification. Primary 31C35, 60J50; Secondary 31B05, 60J45, $60 \mathrm{~J} 65$.

Key words and phrases. Martin boundary, harmonic functions, parabolic functions, Brownian motion, $h$-transforms.

The first author was supported in part by NSF grant DMS-9700721.

The second author was supported in part by a grant from NSERC. A portion of this research took place during his stay at the Fields Institute. 
transition probabilities $P\left(\dot{X}_{u} \in(d y,-d u) \mid \dot{X}_{t} \in(d x,-d t)\right)$ depend not only on $u-t$, but on the values of $t$ and $u$ as well.

Next suppose that $c>0$ is a constant and consider $\dot{X}$ conditioned to be at $(c k, 0,-k)$ at time $k$ and to not leave $D \times \mathbb{R}$ before time $k$. In the limit, as $k \rightarrow \infty$, we obtain a process whose spatial component escapes to " $+\infty$ " within $D$ at rate $c$. The first coordinate of the space process is a one-dimensional Brownian motion with drift $c$. This conditioned space-time Brownian motion is time-homogeneous and its lifetime is infinite.

The domain in our example, a strip, seems to be typical and we would expect that many domains have the property stated in the following problem.

(1.1) Problem. Find necessary and sufficient conditions of a geometric nature in $D$ such that for every minimal parabolic function $h$ in $\dot{D}$, the corresponding $h$ transform of the space-time Brownian motion is time-homogeneous if and only if its lifetime is a.s. infinite.

Another source of motivation may be explained in purely analytic language. Recall the domain of our first example, $D=\left\{\left(x^{1}, x^{2}\right) \in \mathbb{R}^{2}:\left|x^{2}\right|<1\right\}$. Consider a minimal positive harmonic function $h(x), x \in D$. Let $g(x, t)=h(x)$ for all $x \in D$ and $t \in \mathbb{R}$. Evidently, $g$ is a parabolic function, and we may therefore identify every harmonic function with a parabolic function. Since $h$ is minimal harmonic, it corresponds to a minimal Martin boundary point $y$ of $D$. Suppose that $y$ is also a Euclidean boundary point, say, $y=(1,1)$. Then $g$ is not minimal as a parabolic function, i.e., it is a mixture of different parabolic functions. An easy probabilistic justification can be based on the fact that Brownian motion conditioned by $h$ has a random lifetime. Thus the space-time Brownian motion conditioned by $g$ is a mixture of processes conditioned to exit $D$ through $y$ at different times $s$, i.e., a mixture of $g_{s}$-transforms for different parabolic functions $g_{s}$. However, if $y$ is the point at " $+\infty$ ", then $g$ is minimal in the space of parabolic functions. While not completely obvious, this is simple to show directly, and also follows from our main result, Theorem 1.3 below. Our informal discussion suggests that in many domains, a minimal harmonic function is also minimal in the space of parabolic functions if and only if it corresponds to a "point at infinity." We propose the following problem.

(1.2) Problem. Determine which minimal harmonic functions are minimal in the space of parabolic functions.

We are not able to give a complete answer to either of the two problems but we hope that our main result, Theorem 1.3 below, will shed light on both.

We proceed with a rigorous presentation of our results. We start with a review of basic definitions and facts concerning Martin boundaries and conditioned Brownian motion. Let $D$ be a Euclidean domain, that is, an open connected subset of $\mathbb{R}^{d}$ for some $d \geq 2$. We will consider the domain $\dot{D} \stackrel{\text { df }}{=} D \times(-\infty, 0) \subset \mathbb{R}^{d+1}$. Let $G(x, y)=G_{D}(x, y)$ and $\dot{G}(u, v)=\dot{G}_{\dot{D}}(u, v)$ be the Green functions for $(1 / 2) \Delta$ on $D$ and for the heat operator $(1 / 2) \Delta-\partial / \partial t$ on $\dot{D}$ where $\Delta$ is the Laplace operator (see 1.VII.1 and 1.XVII.4 of [Db]). Thus $G: D \times D \rightarrow(0, \infty]$ and $\dot{G}: \dot{D} \times \dot{D} \rightarrow[0, \infty]$. For $u=(x, s) \in \dot{D}$ and $v=(y, s-t) \in \dot{D}$ we have that

$$
\dot{G}(u, v)= \begin{cases}p_{t}(x, y), & \text { for } t>0, \\ 0, & \text { for } s<t \leq 0,\end{cases}
$$


where $p_{t}=p_{t}^{D}$ is the heat kernel on $D$ (that is, the transition function for Brownian motion killed upon leaving $D$ ). Note that this formula can also be used to define $\dot{G}((x, s), v)$ when $s=0$. A function $h: D \rightarrow[0, \infty)$ is harmonic if $\Delta h=0$ on $D$. A function $g: \dot{D} \rightarrow[0, \infty)$ is parabolic if it solves the heat equation

$$
\frac{\partial g}{\partial t}=\frac{1}{2} \Delta_{x} g
$$

in $\dot{D}$. In this case, it is superparabolic as well; that is,

$$
g(x, s) \geq \int g(y, s-t) p_{t}(x, y) d y
$$

for every $(x, s) \in \dot{D}$ and $t>0$. We may extend $g$ by letting

$$
g(x, 0) \stackrel{\mathrm{df}}{=} \lim _{t \downarrow 0} \int g(y,-t) p_{t}(x, y) d y
$$

(the limit is easily seen to be monotone). Fix some $x_{0} \in D$. We say that $g$ is admissible if $g\left(x_{0}, 0\right)<\infty$.

Now recall the definitions of the Martin boundary in the elliptic and parabolic contexts (1.XII.3 and 1.XIX.3 of [Db]). Let

$$
K(x, y) \stackrel{\text { df }}{=} \frac{G(x, y)}{G\left(x_{0}, y\right)}
$$

for $x, y \in D$. Then, up to homeomorphism, there is a unique metrizable compactification $D^{M}$ of $D$ such that

(i) the function $K(\cdot, \cdot)$ may be extended continuously to $D \times\left(D^{M} \backslash\left\{x_{0}\right\}\right)$;

(ii) $K(\cdot, x) \equiv K(\cdot, y)$ if and only if $x=y$.

The set $\partial^{M} D \stackrel{\text { df }}{=} D^{M} \backslash D$ is called the Martin boundary of $D$. For $z \in \partial^{M} D$ and $y_{k} \in D$, we have $y_{k} \rightarrow z$ if and only if $K\left(x, y_{k}\right) \rightarrow K(x, z)$ for every $x \in D$. A harmonic function $h>0$ is said to be minimal if, whenever $h^{\prime}>0$ is harmonic, and $h^{\prime} \leq h$, it follows that $h^{\prime}=c h$ for some constant $c$. A point $z \in \partial^{M} D$ is said to be minimal if $K(\cdot, z)$ is minimal. For every $h>0$ harmonic, there is a unique measure $\mu$, concentrated on the set $\partial_{0}^{M} D$ of minimal points of $\partial^{M} D$, such that

$$
h(x)=\int_{\partial_{0}^{M} D} K(x, z) \mu(d z),
$$

for every $x \in D$ (see 1.XII.9 of [Db]).

Now define $\dot{K}$ on $\dot{D} \times \dot{D}$ by

$$
\begin{aligned}
\dot{K}((x, s),(y, t)) & \stackrel{\mathrm{df}}{=} \frac{\dot{G}((x, s),(y, t))}{\dot{G}\left(\left(x_{0}, 0\right),(y, t)\right)} \\
& = \begin{cases}p_{s-t}(x, y) / p_{-t}\left(x_{0}, y\right), & t<s<0, \\
0, & s \leq t<0 .\end{cases}
\end{aligned}
$$

Then up to homeomorphism, there is a unique metrizable compactification $\dot{D}^{M}$ of $\dot{D}$ with the following properties:

(i) the function $\dot{K}$ has an extension to $\dot{D} \times \dot{D}^{M}$ such that for each $(x, s) \in \dot{D}$, the function $\dot{K}((x, s), \cdot)$ is finite valued and continuous on $\dot{D}^{M} \backslash\{(x, s)\}$;

(ii) $\dot{K}(\cdot, u)=\dot{K}(\cdot, v)$ if and only if $u=v$. 
We call $u$ the pole of $\dot{K}(\cdot, u)$. We write $\partial^{M} \dot{D} \stackrel{\text { df }}{=} \dot{D}^{M} \backslash \dot{D}$ and call it the Martin boundary of $\dot{D}$ (or the parabolic Martin boundary of $D$ ). Again we have that, for $z \in \partial^{M} \dot{D}$ and $\left(y_{k}, t_{k}\right) \in \dot{D},\left(y_{k}, t_{k}\right) \rightarrow z$ if and only if $\dot{K}\left((x, t),\left(y_{k}, t_{k}\right)\right) \rightarrow$ $\dot{K}((x, t), z)$ for every $(x, t) \in \dot{D}$. Every $\dot{K}(\cdot, z)$ is admissible (see 1.XIX.3.1 of $[\mathrm{Db}])$.

We denote by $\dot{0}$ the unique point of $\partial^{M} \dot{D}$ for which $K(\cdot, \dot{0}) \equiv 0$. It is unique by (ii) and exists as the limit of some subsequence of $\left(x_{0}, 1 / n\right)$. A point $z \in \partial^{M} \dot{D}$ is minimal if $\dot{K}(\cdot, z)$ is minimal as a parabolic function, and $\dot{K}\left(\left(x_{0}, 0\right), z\right)=1$. The set of minimal points is denoted by $\partial_{0}^{M} \dot{D}$. The integral representation of admissible parabolic functions as

$$
g(x, t)=\int_{\partial_{0}^{M} \dot{D}} \dot{K}((x, t), z) \mu(d z)
$$

is entirely analogous to that of the harmonic setting (see 1.XIX.7 of [Db]).

Let $(\Omega, \mathcal{F})$ be a measurable space with $X: \Omega \times[0, \infty) \rightarrow \mathbb{R}^{d} \cup\{\delta\}$ a stochastic process. We use the notation $X_{t}$ and $X(t)$ interchangeably. $P^{x}$ is a probability measure under which $X$ is a standard $d$-dimensional Brownian motion started from $x$, and killed upon leaving $D$. We write $E^{x}$ for the corresponding expectation. In particular, $\delta$ is a cemetery point adjoined to $D, X$ is continuous on a random time interval $[0, \zeta)$, and $X_{t}=\delta$ for $t \geq \zeta$.

Let $\tau_{t}=\tau_{0}-t$ be a process measuring absolute time, and write $\dot{X}_{t}=\left(X_{t}, \tau_{t}\right)$. By enlarging $\Omega$ if necessary, we may suppose that for each $s \leq 0$, there are probability measures $P^{x, s}$ under which $X$ has the same law as under $P^{x}$, and $\tau_{0}=s$. That is, $\left\{\dot{X}_{t}, t \geq 0\right\}$ is a space-time Brownian motion starting from $(x, s)$.

If $h: D \rightarrow(0, \infty]$ is a superharmonic function, then

$$
p_{t}^{h}(x, y) \stackrel{\text { df }}{=} \frac{h(y) p_{t}(x, y)}{h(x)}
$$

is the transition function of a Markov process $X^{h}$, called an $h$-transform, or conditioned Brownian motion. We write $P_{h}^{x}$ and $E_{h}^{x}$ for the corresponding probability measure, and its expectations. By convention, $h$ is taken to vanish at $\delta$. If $x \in D^{M}$, $x \neq x_{0}$, then we write $X^{x}$ for $X^{K(\cdot, x)}$. If $h=\int_{\partial_{0}^{M}} K(\cdot, z) \mu(d z)$ is harmonic, then

$$
P_{h}^{x}=\frac{1}{h(x)} \int_{\partial_{0}^{M}} K(x, z) P_{z}^{x} \mu(d z) .
$$

The paths of $X^{h}$ converge a.s. to points of the minimal Martin boundary, at their lifetimes (see 3.III.1 of [Db], or section 7.2 of Pinsky ([P], 1995)).

Similarly, if $g: \dot{D} \rightarrow[0, \infty]$ is a superparabolic function, then

$$
\dot{p}_{t}^{g}((x, s),(y, s-t)) \stackrel{\text { df }}{=} \frac{g(y, s-t) p_{t}(x, y)}{g(x, s)}
$$

is the transition function for a Markov process $\dot{X}^{g}$ taking values in $\dot{D} \cup\{\delta\}$ (actually in $\{\delta\} \cup\{u \in \dot{D} ; g(u)>0\}$ ) that we call a conditioned space-time Brownian motion. We will use $P_{g}^{x, s}$ to denote a probability measure under which $\dot{X}^{g}$ has this transition function and starts from $(x, s)$. We write $X^{g}$ for the spatial component of $\dot{X}^{g}$ (with 
$X_{t}^{g}=\delta$ for $\left.t \geq \zeta\right)$, and note that

$$
\dot{X}_{t}^{g}= \begin{cases}\left(X_{t}^{g}, \tau_{t}\right) \in \dot{D}, & \text { for } t<\zeta, \\ \delta & \text { for } t \geq \zeta .\end{cases}
$$

We will also refer to $X^{g}$ as a $g$-transform. This abuse should cause no confusion, as it is easy to check that if $h$ is superharmonic and we define a superparabolic function $g$ by $g(x, t)=h(x)$, then $X^{h}=X^{g}$. If $u \in \dot{D}^{M}$, then we write $\dot{X}^{u}, X^{u}$, $P_{u}^{x, s}$ instead of $\dot{X}^{K(\cdot, u)}$, etc. Strictly speaking, the above formulae hold under $P_{g}^{x, s}$ only for $s<0$, but by taking $X_{0}^{g}=x$ under $P_{g}^{x, 0}$, we obtain extensions valid for $s=0$ as well, provided $g$ is admissible. If $g$ is actually parabolic, then each $g$-process approaches the one-point boundary of $\dot{D}$ at its lifetime $\zeta$ (2.X.12 of $[\mathrm{Db}])$, in other words, it eventually leaves every compact subset of $\dot{D}$. In the Martin topology, the paths of $\dot{X}$ converge at their lifetimes, to points of the minimal parabolic Martin boundary, and the measures $P_{g}^{x, s}$ can be represented in terms of the $P_{u}^{x, s}$, for $u \in \partial_{0}^{M} \dot{D}$, just as in the harmonic setting.

For $\left(x^{1}, x^{2}, \ldots, x^{d}\right) \in \mathbb{R}^{d}$ let $\widetilde{x}=\left(x^{1}, x^{2}, \ldots, x^{d-1}\right)$. We will restrict our attention to "tubes" with variable width. For a non-negative function $f: \mathbb{R} \rightarrow \mathbb{R}$, let

$$
D_{f} \stackrel{\text { df }}{=}\left\{x \in \mathbb{R}^{d}:|\widetilde{x}|<f\left(x^{d}\right)\right\} .
$$

We will always assume that $f$ is strictly positive on $(a, b)$ for some $-\infty \leq a<b \leq \infty$ and equal to 0 on $(-\infty, a] \cup[b, \infty)$. We will focus on domains $D_{f}$ corresponding to functions $f$ which are Lipschitz on $(a, b)$ (the function may have a jump at $a$ or $b$ ). If $f$ is Lipschitz and $b=\infty$, then each sequence $x_{k}$ of points in $D_{f}$ such that $x_{k}^{d} \rightarrow \infty$ converges in the Martin topology to a point (the same for all such sequences) which we will denote as $\infty$. The proof of this claim is easy - it may be based on the boundary Harnack principle. The same result should be true for all functions $f$ (not necessarily Lipschitz) but we do not see an obvious argument. An analogous remark applies to $-\infty$. Any positive harmonic function $h$ corresponding to $\infty \in \partial^{M} D_{f}$ vanishes on $\left\{x \in \partial D_{f}: x^{d}<b\right\}$ and, moreover, $h(x) \rightarrow 0$ when $x^{d} \rightarrow-\infty$.

Let $\Lambda_{s}=\left\{x \in D_{f}: x^{d}=s\right\}$. The stopping time inf $\left\{t>0: X_{t} \in A\right\}$ will be denoted $T(A)$. We write $\tau(A)$ for the absolute time $\tau_{T(A)}=\tau_{0}-T(A)$.

Recall that a harmonic function $h$ is identified with a parabolic function by letting $h(x, t)=h(x)$.

(1.3) Theorem. Suppose that $b=\infty$ and $f$ is a function which is Lipschitz on $(a, b)$ and such that

$$
\limsup _{v \rightarrow \infty} f(v)<\infty
$$

and

$$
\int_{u}^{\infty} f(v) d v=\infty
$$

for all $u<\infty$. Let $h$ be the minimal harmonic function corresponding to $\infty \in$ $\partial_{0}^{M} D_{f}$. Fix some $x_{0} \in D_{f}$.

(i) Suppose that either

(a) $\int_{a}^{\infty} f^{3}(v) d v<\infty$ or 
(b) the Lipschitz constant of $f$ is sufficiently small (it will suffice to assume that it is less than the $\lambda$ in (iv) of Theorem 1.6) and $\int_{u}^{\infty} f^{3}(v) d v<\infty$ for some $u<\infty$.

Each one of assumptions (a) or (b) implies (A)-(D) below.

(A) For some function $g:(a, \infty) \rightarrow(-\infty, 0]$ with $\lim _{u \rightarrow \infty} g(u)=-\infty$, we have the following. For each $s \in \mathbb{R}$ there is a minimal point $z_{s} \in \partial_{0}^{M} \dot{D}_{f}$, which is the limit of all sequences $\left(x_{k},\left(g\left(x_{k}^{d}\right)-s_{k}\right) \wedge 0\right)$ with $x_{k}^{d} \rightarrow \infty$ and $s_{k} \rightarrow s$.

(B) If $s_{1} \neq s_{2}$, then $z_{s_{1}} \neq z_{s_{2}}$.

(C) Let $h_{s}$ denote a minimal parabolic function with pole at $z_{s}$. Then $h=$ $\int_{\mathbb{R}} h_{s} \mu(d s)$ for some measure $\mu$ which charges all non-degenerate intervals. In particular, $h$ is not minimal in the space of parabolic functions on $\dot{D}_{f}$.

(D) Let $s \in \mathbb{R}$ and $(x, t) \in \dot{D}$. The process $\dot{X}$ is not time-homogeneous under $P_{z_{s}}^{x, t}$. In fact, $g(u)-\tau\left(\Lambda_{u}\right) \rightarrow s$ as $u \rightarrow \infty P_{z_{s}}^{x, t}$-a.s. Hence, $\lim _{u \rightarrow \infty}\left(T\left(\Lambda_{u}\right)+g(u)\right)$ exists $P_{h}^{x}$-a.s.

(ii) If $\int_{u}^{\infty} f^{3}(v) d v=\infty$ for all $u<\infty$, then $h$ is minimal in the space of parabolic functions on $\dot{D}_{f}$.

(1.5) Remarks. The lifetime of Brownian motion conditioned by $h$ is infinite if and only if $\int_{u}^{\infty} f(v) d v=\infty$ for all $u<\infty$, according to Theorem 1.6 below. If this condition is not satisfied, the function $h$ is not minimal as a parabolic function (see the discussion preceding Problem 1.2).

The proof of Theorem 1.3 hinges on estimates of the variance of $h$-path lifetimes. Since the estimates may have some independent interest, we state them as Theorem 1.6 below.

Several authors have addressed the problem of when, given a domain $D \subset \mathbb{R}^{d}$, there is a constant $c=c(D)<\infty$ such that for any $x \in D$ and any positive harmonic function $h$ in $D$ we have $E_{h}^{x} \zeta<c$. The pioneering work was done by Cranston and McConnell ([CM], 1983) and Cranston ([C], 1985). The existence of the finite upper bound $c$ is known for a wide class of domains; see, e.g., Bañuelos and Davis ([BD], 1992) or Bass and Burdzy ([BB2], 1992) and references therein. Higher moments of $h$-path lifetimes have been studied by Davis ([Dv], 1988), Davis and Zhang ([DZ], 1994) and Zhang ([Z], 1996).

Chris Rogers has pointed out to us that a related equivalence, between nonminimality and the variance of hitting times, has been established in the context of one-dimensional diffusions. There, the speed measure and coupling can be used to give a simple proof. See Rogers ([Rg], 1988), which synthesizes earlier work of Fristedt and Orey ([FO], 1978), Küchler and Lunze ([KL], 1980), and Rösler ([Rs], 1979).

Recall that we are concerned with functions $f$ which are strictly positive and Lipschitz on $(a, b)$ and equal to 0 on $(-\infty, a] \cup[b, \infty)$. Our next result holds for all functions $f$ which are Lipschitz on $(a, b)$. However, in order to simplify the notation we will prove it only in the case when $f$ is Lipschitz with the constant equal to 1 , i.e., from now on we will assume that $|f(u)-f(v)| \leq|u-v|$ for $u, v \in(a, b)$. Fix some $s_{0} \in(a, b)$ and define $s_{k}$ inductively by $s_{k+1}=s_{k}+f\left(s_{k}\right) / 2$ for $k \geq 0$ and $s_{k-1}=s_{k}-f\left(s_{k}\right) / 2$ for $k \leq 0$. If $s_{k} \geq b$ for some $k$, then we redefine $s_{j}$ for $j \geq k$ and we let $s_{j}=b$ for all $j \geq k$. A similar remark applies to the case when $s_{k} \leq a$. Note that it may happen that $s_{k}<b$ for all $k>0$ and/or $s_{k}>a$ for all $k<0$. However, we always have $\lim _{k \rightarrow \infty} s_{k}=b$ and $\lim _{k \rightarrow-\infty} s_{k}=a$. Let 
$k_{f}=\inf \left\{k: s_{k}=b\right\}$ and recall that $\Lambda_{s_{k}}=\left\{x \in D_{f}: x^{d}=s_{k}\right\}$. Let $D_{j}$ be the component of $D_{f} \backslash \Lambda_{s_{j}}$ which contains points $x$ with $x^{d}<s_{j}$.

(1.6) Theorem. Let $h$ be a positive harmonic function in $D_{f}$ which vanishes on $\left\{x \in \partial D: x^{d}<b\right\}$. If $b=\infty$, then $h$ corresponds to $\infty \in \partial_{0}^{M} D_{f}$. In the following statements, $x$ ranges over the elements of $D_{f}$ with $x^{d}<b-f(b-)$ (here $\infty-\infty=\infty)$.

(i) For some $c_{1}, c_{2} \in(0, \infty)$,

$$
c_{1} \int_{x^{d}}^{b} f(v) d v \leq E_{h}^{x} \zeta \leq c_{2} \int_{x^{d}}^{b} f(v) d v .
$$

(ii) If $\int_{x^{d}}^{b} f(v) d v=\infty$, then $\zeta=\infty P_{h}^{x}$-a.s.

(iii) If $\zeta<\infty P_{h}^{x}$-a.s., then for some $c_{3}, c_{4} \in(0, \infty)$,

$$
c_{3} \int_{x^{d}}^{b} f^{3}(v) d v \leq \operatorname{Var}_{h}^{x} \zeta \leq c_{4} \int_{a}^{b} f^{3}(v) d v .
$$

(iv) There exists $\lambda>0$ such that if the Lipschitz constant of $f$ is less than $\lambda$, then

$$
\operatorname{Var}_{h}^{x} \zeta \leq c_{5} \int_{x^{d}}^{b} f^{3}(v) d v
$$

(v) If $\int_{x^{d}}^{b} f^{3}(v) d v=\infty$, then for each $c_{6}<\infty$ and $c_{7}>0$ there is a $k_{0}<\infty$ such that for all $k>k_{0}$ and $u \in \mathbb{R}$,

$$
P_{h}^{x}\left(T\left(\Lambda_{s_{k}}\right) \in\left(u, u+c_{6}\right)\right)<c_{7} .
$$

(1.10) Remarks. (i) The constants $c_{j}$ in Theorem 1.6 depend only on the dimension $d$ and the Lipschitz constant of $f$. However, the proof will be given only in the case when the Lipschitz constant of $f$ is equal to 1 so all the constants in Section 2 will depend only on dimension $d$.

(ii) The bound (1.9) holds for $d \geq 4$ without any assumptions on the value of the Lipschitz constant of $f$ but it does not hold without such an assumption for $d<4$. We are not going to prove the latter. It essentially follows from a theorem of Davis and Zhang ([DZ], 1994).

(iii) We can give a meaning to (1.8) and (1.9) even if $\zeta=\infty P_{h}^{x}$-a.s. Note that in such a case we necessarily have $b=\infty$ (see (1.7)). For all $k<\infty$ and $x \in D_{f}$ such that $x^{d}<s_{k}$,

$$
\operatorname{Var}_{h}^{x} T\left(\Lambda_{s_{k}}\right)<c_{4} \int_{a}^{b} f^{3}(v) d v
$$

with the same constant $c_{4}$ as in (1.8). This and the analogous modification of (1.9) can be proved by applying the theorem to the function $\widetilde{f}(v) \stackrel{\text { df }}{=} f(v) \mathbf{1}_{\left(-\infty, s_{k}\right)}(v)$.

(iv) In the two-dimensional case, part (i) of Theorem 1.6 is due to Xu ([X], 1990). This was generalized by Bañuelos and Davis ([BD], 1992).

(v) Suppose that $d=2$, the Lipschitz constant of $f$ is small and let $\rho$ be the supremum of areas of discs contained in $D_{f}$. Then (1.7) and (1.9) imply that $\operatorname{Var}_{h}^{x} \zeta \leq c_{1} \rho E_{h}^{x} \zeta$. Davis ([Dv], 1988) discovered this inequality and proved that it holds for all simply connected planar domains $D$ provided $h$ is a minimal positive harmonic function or a Green function.

We would like to thank Rodrigo Bañuelos, Rich Bass and Burgess Davis for some very useful discussions of $h$-path lifetimes. 


\section{Moments of $h$-TRANSFORM LifEtimeS}

This section contains the proof of Theorem 1.6. We start with a short review of some useful facts about $h$-processes. The proofs may be found in $[\mathrm{Db}]$ and in a paper of Meyer, Smythe and Walsh ([MSW], 1972).

Let $D \subset \mathbb{R}^{d}$ be a Greenian domain and $h$ be a positive superharmonic function in $D$. Suppose that $M$ is a closed subset of $D$ and let $L=\sup \left\{t<\zeta: X_{t} \in M\right\}$ be the last exit time from $M$. Let

$$
\begin{aligned}
& Y_{1}(t)=X(t), \quad t \in(0, T(M)), \\
& Y_{2}(t)=X(T(M)+t), \quad t \in(0, \zeta-T(M)), \\
& Y_{3}(t)=X(t), \quad t \in(0, L), \\
& Y_{4}(t)=X(L+t), \quad t \in(0, \zeta-L), \\
& Y_{5}(t)=X(\zeta-t), \quad t \in(0, \zeta) .
\end{aligned}
$$

Under $P_{h}^{x}$, each process $Y_{k}$ is an $h_{k}$-transform in a domain $D_{k}$, where $D_{1}=D_{4}=$ $D \backslash M$ and $D_{2}=D_{3}=D_{5}=D$. Moreover, $h_{1}=h_{2}=h$. The function $h_{3}$ is a potential supported by $\partial M$. The function $h_{4}$ is harmonic and has the boundary values 0 on $\partial M$ and the same boundary values as $h$ on $\partial D \backslash \partial M$. The function $h_{5}$ is the Green function $G_{D}(x, \cdot)$ if $x \in D$, or a harmonic function with a pole at $x$ if $x \in \partial D$.

If $\mu(d y)$ is the $P^{x}$-distribution of $X(T(M))$, then the $P_{h}^{x}$-distribution of this random variable is $\mu(d y) h(y) / h(x)$.

(2.1) Lemma (Brownian scaling). Suppose $h$ is a positive superharmonic function in a domain $D \subset \mathbb{R}^{d}$ and $x \in D^{M}$. For a fixed $a \in(0, \infty)$ let

$$
\begin{aligned}
D_{a} & \stackrel{\mathrm{df}}{=}\left\{y \in \mathbb{R}^{d}: y / a \in D\right\}, \\
h_{a}(y) & \stackrel{\mathrm{df}}{=} h(y / a) \quad \text { for } y \in D_{a}, \\
x_{a} & \stackrel{\mathrm{df}}{=} a x, \\
X_{t}^{a} & \stackrel{\mathrm{df}}{=} a X_{t / a^{2}} \quad \text { for } t \geq 0 .
\end{aligned}
$$

If $X$ has the distribution $P_{h}^{x}$, then $X^{a}$ has the distribution $P_{h_{a}}^{x_{a}}$.

Proof. The lemma follows immediately from the scaling properties of Brownian motion and superharmonic functions.

A domain $D \subset \mathbb{R}^{d}, d \geq 2$, is called a Lipschitz domain if for every $x \in \partial D$ there is a neighborhood $U_{x}$ of $x$, an orthonormal coordinate system $C S_{x}$ and a Lipschitz function $f_{x}: \mathbb{R}^{d-1} \rightarrow \mathbb{R}$ with constant $\lambda$ (independent of $x$ ) such that $\partial D \cap U_{x}$ is a part of the graph of $f_{x}$ in $C S_{x}$. Note also that the index on any constant $c_{1}, c_{2}, \ldots$ is local in nature. That is, new results or sections of proofs will start numbering their constants with $c_{1}$ as well.

(2.2) Lemma (Boundary Harnack principle). (a) Suppose $f: \mathbb{R}^{d-1} \rightarrow \mathbb{R}$ is a Lipschitz function with constant $\lambda>0,|f(x)| \leq 1$ for all $x \in \mathbb{R}^{d-1}$, and let

$$
\begin{aligned}
D & =\left\{x \in \mathbb{R}^{d}:|\widetilde{x}|<1, f(\widetilde{x})<x^{d}<2\right\}, \\
D_{1} & =\left\{x \in D:|\widetilde{x}|<1 / 2, x^{d}<3 / 2\right\} .
\end{aligned}
$$


There exists $c_{1}>0$ which depends on $\lambda$ but otherwise does not depend on $f$ such that for all $x, y \in D_{1}$ and all positive harmonic functions $g, h$ in $D$ which vanish continuously on $\left\{z \in \partial D: z^{d}=f(\widetilde{z})\right\}$ we have

$$
\frac{g(x)}{g(y)} \geq c_{1} \frac{h(x)}{h(y)} .
$$

(b) Suppose $D$ is a Lipschitz domain, $Q$ is a compact set and $A$ is an open set such that $Q \cap \bar{D} \subset A$. There exists $c_{2}>0$ such that for all $x, y \in Q \cap D$ and all positive harmonic functions $g, h$ in $D$ which vanish continuously on $\partial D \cap A$ we have

$$
\frac{g(x)}{g(y)} \geq c_{2} \frac{h(x)}{h(y)} .
$$

For the first proofs of the boundary Harnack principle, see Ancona ([An], 1978), Dahlberg ([Dg], 1977) and Wu ([W], 1978). Stronger versions of the result may be found in Bass and Burdzy ([BB1], 1991) or Bañuelos, Bass and Burdzy ([BBB], 1991).

Part (a) of Lemma 2.2 holds (with the same $c_{1}$ ) in domains which may be obtained from $D$ by scaling.

When applying the boundary Harnack principle we will sometimes leave it to the reader to find the right choice of $D$ and $D_{1}$ or $D, A$ and $Q$.

(2.3) Lemma. Suppose $D$ is a domain, $D_{1}$ is a Lipschitz subdomain of $D, Q$ is a compact set, $A$ is an open set such that $Q \cap \bar{D} \subset A, A \cap D \subset D_{1}$, and $M$ is a Borel subset of $D \backslash A$. Assume that $h$ is a positive superharmonic function in $D$ which vanishes on $\partial D \cap A$ and is harmonic in $D_{1}$. Then

$$
P_{h}^{x}(T(M)<\infty) \leq c_{1} P_{h}^{y}(T(M)<\infty)
$$

for all $x, y \in Q \cap D$. The constant $c_{1}$ depends only on $D_{1}, Q$ and $A$.

Proof. The function

$$
x \rightarrow E^{x}[T(M)<T(\partial D), h(X(T(M)))]
$$

is positive and harmonic in $A \cap D$ and the same is true for $x \rightarrow h(x)$. Let $D_{2}$ be a Lipschitz subdomain of $A \cap D$ which contains $Q$. By the boundary Harnack principle, Lemma 2.2(b), applied in $D_{2}$,

$$
\begin{aligned}
P_{h}^{x}(T(M)<\infty) & =\frac{1}{h(x)} E^{x}[T(M)<T(\partial D), h(X(T(M)))] \\
& \leq c_{2} \frac{1}{h(y)} E^{y}[T(M)<T(\partial D), h(X(T(M)))] \\
& =c_{2} P_{h}^{y}(T(M)<\infty) .
\end{aligned}
$$

(2.4) Lemma. Suppose $D$ is a domain and for each $k=1,2$,

(i) $D_{k}$ is a subdomain of $D$,

(ii) $A_{k} \stackrel{\text { df }}{=} \partial D_{k} \cap D$,

(iii) $V_{k}$ is an open set and $Q_{k}$ is a compact set such that $Q_{k} \cap \bar{D} \subset V_{k}$ and $\bar{V}_{k} \cap D \subset D_{k}$,

(iv) $\left(D_{1} \cup V_{1}\right) \cap\left(D_{2} \cup V_{2}\right)=\emptyset$, 
(v) there is a $c_{k}>0$ such that for all $x, y \in Q_{k} \cap D$ and all positive harmonic functions $f, g$ in $D_{k}$ which vanish on $V_{k} \cap \partial D$ we have

$$
\frac{f(x)}{f(y)} \geq c_{k} \frac{g(x)}{g(y)}
$$

Assume that $x_{1}, x_{2} \in \overline{Q_{1} \cap D}$ and $h_{1}, h_{2}$ are positive superharmonic functions in $D$ which vanish continuously on $\partial D \backslash V_{2}$ and are harmonic in $D \backslash Q_{2}$. Let $T_{1} \stackrel{\mathrm{df}}{=} T\left(A_{1}\right)$ and let $T_{2}$ be the last exit time from $A_{2}$. The distributions of $\left\{X_{t}, t \in\left[T_{1}, T_{2}\right]\right\}$ under $P_{h_{1}}^{x_{1}}$ and $P_{h_{2}}^{x_{2}}$ are mutually absolutely continuous and their Radon-Nikodym derivative is bounded below by $c_{1} c_{2}$.

Proof. We will consider only the case when $x_{k} \in Q_{1} \cap D$ and $h_{k}(\cdot)=G_{D}\left(\cdot, y_{k}\right)$ for some $y_{k} \in Q_{2} \cap D$. Other points $x_{k}$ and functions $h_{k}$ may be treated analogously.

Under $P_{y_{k}}^{x_{k}}$, the process $\left\{X_{t}, t \in\left[T_{1}, \zeta\right]\right\}$ is a $G_{D}\left(\cdot, y_{k}\right)$-process with the initial distribution

$$
\mu_{k}(\cdot) \stackrel{\mathrm{df}}{=} P_{y_{k}}^{x_{k}}\left(X\left(T_{1}\right) \in \cdot\right)=P^{x_{k}}\left(T_{1}<T\left(D^{c}\right), X\left(T_{1}\right) \in \cdot\right) G_{D}\left(\cdot, y_{k}\right) / G_{D}\left(x_{k}, y_{k}\right)
$$

supported on $A_{1}$. For a fixed $z \in A_{1}$, the process $Y_{t} \stackrel{\text { df }}{=} X_{\zeta-t}$ under $P_{y_{k}}^{z}$ has the distribution $P_{z}^{y_{k}}$. If $T_{3}=\inf \left\{t: Y_{t} \in A_{2}\right\}$, then $T_{3}=\zeta-T_{2}$. The process $\left\{Y_{t}, t \in\left[T_{3}, \zeta\right)\right\}$ under $P_{y_{k}}^{z}$ is a $G_{D}(\cdot, z)$-process with the initial distribution

$$
\nu_{k}(\cdot) \stackrel{\mathrm{df}}{=} P^{y_{k}}\left(T\left(A_{2}\right)<T\left(D^{c}\right), X\left(T\left(A_{2}\right)\right) \in \cdot\right) G_{D}(\cdot, z) / G_{D}\left(y_{k}, z\right) .
$$

For a fixed $v \in A_{2}$, the function $y \rightarrow P^{y}\left(T\left(A_{2}\right)<T\left(D^{c}\right), X\left(T\left(A_{2}\right)\right) \in d v\right)$ is positive and harmonic in $D_{2}$ and vanishes on $V_{2} \cap \partial D$ and the same is true for $z \rightarrow G_{D}(v, z)$. By $(2.5)$,

$$
\frac{d \nu_{k}}{d \nu_{3-k}}(v)=\frac{P^{y_{k}}\left(T\left(A_{2}\right)<T\left(D^{c}\right), X\left(T\left(A_{2}\right)\right) \in d v\right) G_{D}(v, z) G_{D}\left(y_{3-k}, z\right)}{G_{D}\left(y_{k}, z\right) P^{y_{3-k}}\left(T\left(A_{2}\right)<T\left(D^{c}\right), X\left(T\left(A_{2}\right)\right) \in d v\right) G_{D}(v, z)} \geq c_{2} .
$$

After reversing time again, we see that the distributions of $X\left(T_{2}\right)$ under $P_{y_{1}}^{z}$ and $P_{y_{2}}^{z}$ have Radon-Nikodym derivative bounded below by $c_{2}$. The process $\left\{X_{t}, t \in\right.$ $\left.\left[T_{1}, T_{2}\right]\right\}$ under $P_{y_{1}}^{z}$ is a mixture of $h$-transforms converging to $w$ with the mixing measure $P_{y_{1}}^{z}\left(X\left(T_{2}\right) \in d w\right)$ and the same remark applies to $P_{y_{2}}^{z}$. Hence, the distributions of $\left\{X_{t}, t \in\left[T_{1}, T_{2}\right]\right\}$ under $P_{y_{1}}^{z}$ and $P_{y_{2}}^{z}$ have a Radon-Nikodym derivative bounded below by $c_{2}$.

We can prove in a similar way that $d \mu_{k}(\cdot) / d \mu_{3-k}(\cdot) \geq c_{1}$. The distributions of $\left\{X_{t}, t \in\left[T_{1}, T_{2}\right]\right\}$ under $P_{y_{1}}^{x_{1}}$ and $P_{y_{2}}^{x_{2}}$ have the Radon-Nikodym derivative bounded below by $c_{1} c_{2}$ because $P_{y_{k}}^{x_{k}}$ is a mixture of the measures $P_{y_{k}}^{z}$ with the mixing measure $\mu_{k}$.

(2.6) Lemma. Suppose that $f: \mathbb{R}^{d-1} \rightarrow \mathbb{R}$ is Lipschitz with constant $\lambda$ and assume that $|f(x)| \leq 1$ for all $x$. Let

$$
D=\left\{x \in \mathbb{R}^{d}:|\widetilde{x}|<1, f(\widetilde{x})<x^{d}<2\right\} .
$$

There exists $c<\infty$ (which may depend on $\lambda$ but does not otherwise depend on $f$ ) such that for every $x \in \bar{D}$ and every positive harmonic function $h$ in $D$

$$
E_{h}^{x} \zeta<c
$$


Proof. The result is essentially due to Cranston ([C], 1985) but we refer the reader to the paper by Bass and Burdzy ([BB2], 1992). Our domain $D$ is a special case of a "twisted Hölder domain" and (2.7) follows from Theorem 1.1 (i), (a), (C) of [BB2]. A direct inspection of its proof shows that $c$ depends only on the volume and diameter of $D$ (under the assumption that $f$ is Lipschitz with constant $\lambda$ ) and these quantities may be bounded independently of the particular form of $f$.

(2.8) Remark. It is not necessary to assume in Lemma 2.6 that $f$ is Lipschitz. It is enough to suppose that $f$ is upper semicontinuous and $f(x)$ is bounded in the $L^{p}$-norm for a suitable $p=p(d)$. This version of the result uses Theorem 1.1 (i), (a), (A) of [BB2] which has a considerably more complicated proof than Theorem 1.1 (i), (a), (C). We feel it would not be fair to ask the reader to go through the former proof in order to check that the constants may be chosen independently of $f$.

(2.9) Lemma. Suppose that $D \subset \mathbb{R}^{d}$ is a domain, $x, y \in \bar{D}$, and for each $v=x, y$ there exist an orthonormal coordinate system $C S_{v}$, a point $z_{v} \in D$, a Lipschitz function $f_{v}$ with constant $\lambda$ and a constant $c_{v}>0$ such that $\left|f_{v}\right| \leq c_{v}$,

$$
\begin{gathered}
D_{v} \stackrel{\mathrm{df}}{=}\left\{z \in D:|\widetilde{z}|<c_{v},-c_{v}<z^{d}<2 c_{v} \text { in } C S_{v}\right\} \\
=\left\{z \in \mathbb{R}^{d}:|\widetilde{z}|<c_{v}, f_{v}(\widetilde{z})<z^{d}<2 c_{v} \text { in } C S_{v}\right\}, \\
z_{v}=\left(0,0, \ldots, 0,3 c_{v} / 2\right) \text { in } C S_{v}, \\
|\widetilde{v}| \leq c_{v} / 2 \text { and } v^{d} \leq 3 c_{v} / 2 \text { in } C S_{v}, \\
D_{x} \cap D_{y}=\emptyset .
\end{gathered}
$$

$$
\text { If } E_{z_{y}}^{z_{x}} \zeta=c_{1} \text {, then }
$$

$$
E_{y}^{x} \zeta \leq c_{2} c_{1}+c_{3}\left(c_{x}^{2}+c_{y}^{2}\right)
$$

where $c_{2}$ and $c_{3}$ depend only on the dimension $d$ and the Lipschitz constant $\lambda$.

Proof. For $v=x, y$ let

$$
\begin{aligned}
& D_{v}^{1}=\left\{z \in D_{v}:|\widetilde{z}|<3 c_{v} / 4, z^{d}<7 c_{v} / 4 \text { in } C S_{v}\right\}, \\
& A_{v}=\partial D_{v}^{1} \cap D, \\
& Q_{v}=\left\{z \in \bar{D}_{v}:|\widetilde{z}| \leq c_{v} / 2, z^{d} \leq 3 c_{v} / 2 \text { in } C S_{v}\right\}, \\
& V_{v}=\left\{z \in \mathbb{R}^{d}:\left|z-Q_{v}\right|<c_{v} / 8\right\} .
\end{aligned}
$$

By the boundary Harnack principle, Lemma 2.2(a), applied in $D_{v}$, assumption (2.5) of Lemma 2.4 holds. Let $T_{1}$ be the first hitting time of $A_{x}$ and let $T_{2}$ be the last exit time from $A_{y}$. By Lemma 2.4,

$$
E_{y}^{x}\left(T_{2}-T_{1}\right) \leq c_{4} E_{z_{y}}^{z_{x}}\left(T_{2}-T_{1}\right) \leq c_{4} E_{z_{y}}^{z_{x}} \zeta .
$$

Lemma 2.6 and Brownian scaling (2.1) imply that

$$
E_{y}^{x} T_{1} \leq c_{5} c_{x}^{2} .
$$

The same lemma and time-reversal show that

$$
E_{y}^{x}\left(\zeta-T_{2}\right) \leq c_{5} c_{y}^{2}
$$

The lemma follows from (2.10)-(2.12). 
We now return to the specific domains, hypotheses, and notation of Theorem 1.6 .

(2.13) Lemma. Assume that $a<s_{j-1}<s_{j}<b$. There exists $c_{1}>0$ such that for every positive harmonic function $h$ in $D_{j}$ which vanishes on $\partial D_{j} \backslash \Lambda_{s_{j}}$ and every $x \in \Lambda_{s_{j-1}}$,

$$
E_{h}^{x} \zeta \geq c_{1} f^{2}\left(s_{j-1}\right)
$$

Moreover, there is a non-negative, non-constant and bounded random variable $Y$ such that for every $j$ and $x \in \Lambda_{s_{j-1}}$, the distribution of $\zeta$ under $P_{h}^{x}$ is stochastically larger than that of $f^{2}\left(s_{j-1}\right) Y$.

Proof. Let $B(y, r)$ denote the ball with center $y$ and radius $r$. Let $c_{2}$ be the expected lifetime of conditioned Brownian motion in $B(0,1)$ starting from 0 and converging to $x \in \partial B(0,1)$. The constant $c_{2}$ is strictly positive and does not depend on $x$ by symmetry. For any harmonic function $g$ in $B(0,1)$, the $g$-process starting from 0 is a mixture of processes conditioned to go to some point of $\partial B(0,1)$ so its expected lifetime is also equal to $c_{2}$. By scaling, the expected lifetime of any Brownian motion conditioned by a harmonic function in $B(y, r)$ and starting from $y$ is equal to $c_{2} r^{2}$.

Let

$$
\begin{aligned}
B_{0} & =B\left(\left(0, \ldots, 0, s_{j-1}+f\left(s_{j-1}\right) / 4\right), f\left(s_{j-1}\right) / 8\right), \\
T_{1} & =\inf \left\{t>T\left(B_{0}\right):\left|X_{t}-X\left(T\left(B_{0}\right)\right)\right|=f\left(s_{j-1}\right) / 16\right\} .
\end{aligned}
$$

Note that $B_{0} \subset D_{j}$. By the strong Markov property applied at $T\left(B_{0}\right)$,

$$
E_{h}^{x} \zeta \geq E_{h}^{x}\left[\left(T_{1}-T\left(B_{0}\right)\right) \mathbf{1}_{\left\{T\left(B_{0}\right)<\infty\right\}}\right]=c_{2}\left(f\left(s_{j-1}\right) / 16\right)^{2} P_{h}^{x}\left(T\left(B_{0}\right)<\infty\right) .
$$

Let $x_{0}=\left(0, \ldots, 0, s_{j-1}\right)$. By Lemma 2.3 , for all $x \in \Lambda_{s_{j-1}}$,

$$
P_{h}^{x}\left(T\left(B_{0}\right)<\infty\right) \geq c_{3} P_{h}^{x_{0}}\left(T\left(B_{0}\right)<\infty\right) .
$$

It is not hard to see that the constant $c_{3}$ may be chosen independently of the particular form of $f$. The probability $P_{h}^{x_{0}}\left(T\left(B_{0}\right)<\infty\right)$ is not less than

$$
P^{x_{0}}\left(T\left(B_{0}\right)<T\left(\partial D_{j}\right)\right) \inf _{y \in B_{0}} h(y) / h\left(x_{0}\right) .
$$

It is elementary to see that $P^{x_{0}}\left(T\left(B_{0}\right)<T\left(\partial D_{j}\right)\right)$ is bounded below and the usual Harnack principle shows that the same is true for $\inf _{y \in B_{0}} h(y) / h\left(x_{0}\right)$. Hence, $P_{h}^{x_{0}}\left(T\left(B_{0}\right)<\infty\right)$ is bounded below by $c_{4}>0$ which together with (2.14) and (2.15) implies

$$
E_{h}^{x} \zeta \geq c_{2}\left(f\left(s_{j-1}\right) / 16\right)^{2} c_{3} c_{4}
$$

It is clear from our proof that $Y$ can be chosen as follows. Let $\widetilde{\zeta}$ be the hitting time of $\partial B(0,1 / 16)$ by a Brownian motion starting from 0 and let $W$ be an independent random variable with $P(W=1)=1-P(W=0)=c_{3} c_{4}$. Then let $Y=W Y^{\prime}$, where $Y^{\prime}=c_{2} \min (\widetilde{\zeta}, 1)$.

(2.16) Lemma. Suppose that $s_{j}<s_{n}$. Let $T_{j}^{1}=T\left(\Lambda_{s_{j}}\right)$ and

$$
\begin{aligned}
& S_{j}^{k}=\inf \left\{t>T_{j}^{k}: X_{t} \in \Lambda_{s_{j-1}} \cup \Lambda_{s_{j+1}}\right\}, \quad k \geq 1, \\
& T_{j}^{k}=\inf \left\{t>S_{j}^{k-1}: X_{t} \in \Lambda_{s_{j}}\right\}, \quad k>1 .
\end{aligned}
$$


There exist $c_{1}<\infty$ and $p<1$ such that for all $k$ and for every positive harmonic function $h$ in $D_{n}$ which vanishes on $\partial D_{n} \backslash \Lambda_{s_{n}}$ and every $x \in D_{n}$

$$
P_{h}^{x}\left(T_{j}^{k}<\infty\right)<c_{1} p^{k}
$$

Moreover, if $i \geq 0, j+i<n$ and $x \in \Lambda_{s_{j+i}}$, then

$$
P_{h}^{x}\left(T_{j}^{k}<\infty\right)<c_{1} p^{k+i} .
$$

Proof. Suppose $s_{k}<s_{k+1} \leq s_{n}$. We have

$$
h(x)=\int_{\Lambda_{s_{k+1}}} h(y) P^{x}\left(X\left(T\left(\Lambda_{s_{k+1}}\right)\right) \in d y\right)
$$

for $x \in \Lambda_{s_{k}}$. The boundary Harnack principle implies that

$$
\frac{P^{x_{1}}\left(X\left(T\left(\Lambda_{s_{k+1}}\right)\right) \in d y\right)}{P^{x_{2}}\left(X\left(T\left(\Lambda_{s_{k+1}}\right)\right) \in d y\right)} \cdot \frac{P^{x_{2}}\left(T\left(\Lambda_{s_{k+1}}\right)<\infty\right)}{P^{x_{1}}\left(T\left(\Lambda_{s_{k+1}}\right)<\infty\right)}<c_{3}<\infty
$$

for $x_{1}, x_{2} \in \Lambda_{s_{k}}$. Let $z_{k}=\left(0, \ldots, 0, s_{k}\right)$. It is easy to see that there is $c_{4}>0$ such that for all $x \in \Lambda_{s_{k}}$ with $|\widetilde{x}|>\left(1-c_{4}\right) f\left(s_{k}\right)$, we have

$$
P^{x}\left(T\left(\Lambda_{s_{k+1}}\right)<\infty\right)<\left(c_{3}^{-1} / 2\right) P^{z_{k}}\left(T\left(\Lambda_{s_{k+1}}\right)<\infty\right) .
$$

This, (2.17) and (2.18) imply that $h(x) \leq h\left(z_{k}\right) / 2$ for $x \in \Lambda_{s_{k}}$ with $|\widetilde{x}|>$ $\left(1-c_{4}\right) f\left(s_{k}\right)$. It follows that the maximum of $h$ on $\Lambda_{s_{k}}$ is attained at a point in the set

$$
A_{k} \stackrel{\mathrm{df}}{=}\left\{x \in \Lambda_{s_{k}}:|\widetilde{x}| \leq\left(1-c_{4}\right) f\left(s_{k}\right)\right\} .
$$

Let $a_{k}$ be the maximum of $h$ over $\Lambda_{s_{k}}$. Since

$$
P^{x}\left(T\left(\Lambda_{s_{k+1}}\right) \leq T\left(\partial D_{n}\right)\right)<c_{5}<1
$$

for $x \in \Lambda_{s_{k}}$, we have $a_{k}<c_{5} a_{k+1}$ assuming $a<s_{k}<s_{k+1}<b$. It follows that $a_{k}<c_{5}^{j} a_{k+j}$. By the Harnack principle, $h(x)>c_{6} a_{k}$ for some $c_{6}>0$ and all $x \in A_{k}$. Let $m$ be so large that $c_{6} c_{5}^{-m}>2$. Then $a_{k}<h(x) / 2$ for all $x \in A_{k+m}$ provided $a<s_{k}<s_{k+m}<b$. We obtain

$$
P_{h}^{x}\left(T\left(\Lambda_{s_{j}}\right)<\infty\right)=\int_{\Lambda_{s_{j}}} \frac{h(y)}{h(x)} P^{x}\left(X\left(T\left(\Lambda_{s_{j}}\right)\right) \in d y\right) \leq 1 / 2
$$

for $x \in A_{j+m}$. Here and later in the proof we assume that $a<s_{j}<s_{j+m}<b$. This assumption could be easily disposed of. We have

$$
P^{z_{k}}\left(T\left(A_{k+1}\right)<T\left(\partial D_{n} \cup \Lambda_{s_{k-1}}\right)\right)>c_{7}>0
$$

and an application of the Harnack principle shows that

$$
P_{h}^{z_{k}}\left(T\left(A_{k+1}\right)<T\left(\Lambda_{s_{k-1}}\right)\right)>c_{8}>0 .
$$

By Lemma 2.3,

$$
P_{h}^{x}\left(T\left(A_{k+1}\right)<T\left(\Lambda_{s_{k-1}}\right)\right)>c_{9}>0
$$

for all $x \in \Lambda_{s_{k}}$. By the strong Markov property applied at the hitting times of $A_{i}$,

$$
P_{h}^{x}\left(T\left(A_{j+m}\right)<T\left(\Lambda_{s_{j}}\right)\right)>c_{9}^{m-1}
$$


for all $x \in \Lambda_{s_{j+1}}$. Let

$$
\begin{aligned}
& U_{1}=\inf \left\{t>T\left(\Lambda_{s_{j+1}}\right): X_{t} \in A_{j+m}\right\}, \\
& U_{2}=\inf \left\{t>T\left(\Lambda_{s_{j+1}}\right): X_{t} \in \Lambda_{s_{j}}\right\}, \\
& U_{3}=\inf \left\{t>U_{1}: X_{t} \in \Lambda_{s_{j}}\right\} .
\end{aligned}
$$

Then (2.19)-(2.21) imply that for $x \in \Lambda_{s_{j}}$

$$
P_{h}^{x}\left(T_{j}^{2}=\infty\right) \geq P_{h}^{x}\left(T\left(\Lambda_{s_{j+1}}\right)<T\left(\Lambda_{s_{j-1}}\right), U_{1}<U_{2}, U_{3}=\infty\right)>c_{9}^{m} / 2>0
$$

for $x \in \Lambda_{s_{j}}$. Both conclusions of the lemma now follow by the repeated application of the strong Markov property at the stopping times $T_{j}^{k}$.

(2.22) Lemma. For all $x_{1} \in D_{f}$ such that $s_{k+1} \leq x_{1}^{d} \leq s_{k+2}$ and $x_{2} \in \Lambda_{s_{k}}$ we have $E_{x_{2}}^{x_{1}} \zeta<c_{1} f^{2}\left(s_{k}\right)$ where $E_{x_{2}}^{x_{1}}$ refers to the conditioned Brownian motion in $D_{f}$.

Proof. We will suppose that $x_{1} \in \Lambda_{s_{k+1}}$. The modifications needed for the general case are obvious.

By Brownian scaling (2.1), we may assume that $f\left(s_{k}\right)=1$ and prove that $E_{x_{2}}^{x_{1}} \zeta<$ $c_{1}$. Note that then $\left|x_{1}^{d}-x_{2}^{d}\right|=1 / 2$.

We have

$$
E_{x_{2}}^{x_{1}} \zeta=c_{2} \int_{D_{f}} \frac{G_{D_{f}}\left(x_{1}, z\right) G_{D_{f}}\left(z, x_{2}\right)}{G_{D_{f}}\left(x_{1}, x_{2}\right)} d z .
$$

In view of Lemma 2.9 it will suffice to prove the lemma for $x_{1} \in \Lambda_{s_{k+1}},\left|\widetilde{x}_{1}\right|<$ $c_{3} f\left(s_{k+1}\right)$, and $x_{2} \in \Lambda_{s_{k}},\left|\widetilde{x}_{2}\right|<c_{3}$ for some $c_{3}<1$. Under this additional assumption, $x_{1}$ and $x_{2}$ may be connected in $D_{f}$ by a Harnack chain of balls of bounded length and this implies that $G_{D_{f}}\left(x_{1}, x_{2}\right)>c_{4}>0$. Hence,

$$
E_{x_{2}}^{x_{1}} \zeta<c_{5} \int_{D_{f}} G_{D_{f}}\left(x_{1}, z\right) G_{D_{f}}\left(z, x_{2}\right) d z
$$

Let

$$
\begin{aligned}
& A_{j}=\left\{z \in D_{f}:\left|z-x_{j}\right|<5,\left|z-x_{3-j}\right|>\left|x_{1}-x_{2}\right| / 2\right\}, \quad j=1,2, \\
& A_{3}=\left\{z \in D_{f}:\left|z-x_{1}\right| \geq 5, z^{d}<s_{k}\right\} \\
& A_{4}=\left\{z \in D_{f}:\left|z-x_{1}\right| \geq 5, z^{d}>s_{k+1}\right\} .
\end{aligned}
$$

Assume for now that $d \geq 3$, and recall that $G(x, y) \stackrel{\text { df }}{=} G_{\mathbb{R}^{d}}(x, y)=c_{6}|x-y|^{2-d}$. For $j=1,2$ we obtain

$$
\begin{gathered}
\int_{A_{j}} G_{D_{f}}\left(x_{1}, z\right) G_{D_{f}}\left(z, x_{2}\right) d z \leq \int_{A_{j}} G\left(x_{1}, z\right) G\left(z, x_{2}\right) d z \\
\quad \leq c_{7} \int_{A_{j}}\left(\left|x_{1}-x_{2}\right| / 2\right)^{2-d}\left|z-x_{j}\right|^{2-d} d z \\
\quad \leq c_{7}\left(\left|x_{1}-x_{2}\right| / 2\right)^{2-d} \int_{0}^{5} r^{2-d} r^{d-1} d r<c_{8}<\infty .
\end{gathered}
$$

Let $x_{0}=\left(0, \ldots, 0, s_{k}\right)$,

$$
\begin{aligned}
\widetilde{D} & =\left\{x \in \mathbb{R}^{d}: x^{d}<s_{k}\right\}, \\
D_{*} & =D_{f} \cup\left\{x \in \mathbb{R}^{d}: x^{d} \in\left(-\infty, s_{k}\right) \cup\left(s_{k+1}, \infty\right)\right\}, \\
M & =\left\{x \in \widetilde{D}:\left|x-x_{1}\right|=4\right\} .
\end{aligned}
$$


The Poisson kernel $K(x)$ in $\widetilde{D}$ with the pole at $x_{0}$ has the form $c_{9}\left|x^{d}-s_{k}\right| /\left|x-x_{0}\right|^{d}$ (1.VIII.9 of $[\mathrm{Db}])$. By the boundary Harnack principle,

$$
G_{D_{*}}\left(x_{1}, x\right) \leq c_{10} K(x)
$$

for $x \in M$ and, therefore, for all $x \in \widetilde{D}$ such that $\left|x-x_{1}\right| \geq 4$, in particular, for $x \in A_{3}$. Hence, for $x \in A_{3}$,

$$
G_{D_{*}}\left(x_{1}, x\right) \leq c_{11}\left|x^{d}-s_{k}\right| /\left|x-x_{0}\right|^{d} \leq c_{11}\left|x-x_{0}\right|^{1-d}
$$

and the same estimate holds for $G_{D_{*}}\left(x_{2}, x\right)$. It follows that

$$
\begin{aligned}
\int_{A_{3}} G_{D_{f}}\left(x_{1}, z\right) G_{D_{f}}\left(z, x_{2}\right) d z & \leq \int_{A_{3}} G_{D_{*}}\left(x_{1}, z\right) G_{D_{*}}\left(z, x_{2}\right) d z \\
& \leq \int_{A_{3}}\left(c_{11}\left|z-x_{0}\right|^{1-d}\right)^{2} d z \\
& \leq c_{12} \int_{2}^{\infty} r^{2(1-d)} r^{d-1} d r<c_{13}<\infty
\end{aligned}
$$

and a similar estimate holds for $A_{4}$. Since $D_{f} \subset A_{1} \cup A_{2} \cup A_{3} \cup A_{4}$, the lemma follows from (2.23)-(2.25).

If $d=2$, an argument similar to the above could be given. In this case, $\widetilde{D}$ should be replaced by a suitable wedge with angle $\alpha<\pi$. The Green function in such a wedge decays like $r^{-\pi / \alpha}$, and this is sufficient to make the bounding integrals finite.

(2.26) Lemma. For $x \in D_{f}$ and $y \in \Lambda_{s_{k}}$, let

$$
g_{x}^{k}(y) d y \stackrel{\text { df }}{=} P_{h}^{x}\left(X\left(T\left(\Lambda_{s_{k}}\right)\right) \in d y\right) .
$$

Then there exist $c_{1}<\infty$ and $c_{2}<1$ such that

$$
\frac{g_{x_{1}}^{n}\left(y_{1}\right)}{g_{x_{1}}^{n}\left(y_{2}\right)} \geq a_{i} \frac{g_{x_{2}}^{n}\left(y_{1}\right)}{g_{x_{2}}^{n}\left(y_{2}\right)}
$$

and

$$
a_{i} \geq 1-c_{1} c_{2}^{i}
$$

for all $i>0$, all $n$, where $x_{1}, x_{2} \in D_{n-i}$ and $y_{1}, y_{2} \in \Lambda_{s_{n}}$.

Proof. A standard application of the boundary Harnack principle in the spirit of Lemma 2.3 shows that (2.27) holds for $i=1$ with some $a_{1}>0$.

Assume that (2.27) holds for all $n$ and for some $i$; we will show that it holds for $i+1$ as well. Let $j=n-i$. By the strong Markov property applied at $T\left(\Lambda_{s_{n-1}}\right)$,

$$
g_{x}^{n}(y)=\int_{\Lambda_{s_{n-1}}} g_{x}^{n-1}(v) g_{v}^{n}(y) d v
$$

for $y \in D_{j-1}$. Now apply Lemma 6.1 of Burdzy, Toby and Williams ([BTW], 1989). Set in that lemma $V=W=\Lambda_{s_{n-1}}$ and $U=\emptyset$, set $f_{1}$ and $f_{2}$ equal to our $g_{x_{1}}^{n-1}$ and $g_{x_{2}}^{n-1}$, set $g_{z}(v)$ equal to our $g_{v}^{n}(z)$, and take $c=a_{i}, d=a_{1}$, and $b=1$. The aforementioned lemma implies that

$$
\frac{g_{x_{1}}^{n}\left(y_{1}\right)}{g_{x_{1}}^{n}\left(y_{2}\right)} \geq a_{i+1} \frac{g_{x_{2}}^{n}\left(y_{1}\right)}{g_{x_{2}}^{n}\left(y_{2}\right)}
$$


for all $y_{1}, y_{2} \in D_{j-1}$, where

$$
a_{i+1}=a_{i}+a_{1}^{2}\left(1-a_{i}\right) .
$$

Hence

$$
1-a_{i+1}=1-a_{i}-a_{1}^{2}\left(1-a_{i}\right)=\left(1-a_{i}\right)\left(1-a_{1}^{2}\right)
$$

and, by induction,

$$
1-a_{i+1} \leq c_{1} c_{2}^{i}
$$

with $c_{2} \stackrel{\mathrm{df}}{=} 1-a_{1}^{2}<1$.

(2.28) Corollary. With the notation of Lemma 2.26,

$$
a_{n-j}^{-1} \geq \frac{g_{x_{1}}^{n}(y)}{g_{x_{2}}^{n}(y)} \geq a_{n-j}
$$

for every $j<n, x_{1}, x_{2} \in D_{j}$ and $y \in \Lambda_{s_{n}}$.

Proof. Let $M$ and $m$ be the supremum and infimum of $g_{x_{1}}^{n}(y) / g_{x_{2}}^{n}(y)$ over $y \in \Lambda_{s_{n}}$. By Lemma 2.26, $m \geq a_{n-j} M$, and

$$
M g_{x_{2}}^{n}(y) \geq g_{x_{1}}^{n}(y) \geq m g_{x_{2}}^{n}(y) .
$$

Integrating with respect to $y$ shows that $M \geq 1 \geq m$, from which the desired conclusion follows.

Proof of Theorem 1.6. (i) We will first prove the lower bound in (1.7).

Suppose that $s_{j_{0}} \leq x^{d}<s_{j_{0}+1}<s_{j_{0}+2}<b$. The other cases are left to the reader. Let $T_{j}=T\left(\Lambda_{s_{j}}\right)$. For each $j>j_{0}+2$ the process $\left\{X_{t}, t \in\left[T_{j-1}, T_{j}\right)\right\}$ under $P_{h}^{x}$ is a conditioned Brownian motion in $D_{j}$ starting from a (random) point in $\Lambda_{s_{j-1}}$ and converging to $\Lambda_{s_{j}}$ at its lifetime. By Lemma 2.13, for $j \in\left[j_{0}+2, k_{f}-1\right]$,

$$
E_{h}^{x}\left(T_{j}-T_{j-1}\right) \geq c_{1} f^{2}\left(s_{j-1}\right)
$$

and, therefore,

$$
E_{h}^{x} \zeta \geq \sum_{j=j_{0}+2}^{k_{f}-1} E_{h}^{x}\left(T_{j}-T_{j-1}\right) \geq \sum_{j=j_{0}+2}^{k_{f}-1} c_{1} f^{2}\left(s_{j-1}\right) .
$$

Since

$$
c_{2} f^{2}\left(s_{j-1}\right)<\int_{s_{j-1}}^{s_{j}} f(v) d v<c_{3} f^{2}\left(s_{j-1}\right),
$$

the sum on the right hand side of (2.29) is bounded below by $c_{4} \int_{s_{j_{0}+1}}^{k_{f}-2} f(v) d v$. Note that

$$
\int_{x^{d}}^{s_{j_{0}+1}} f(v) d v<c_{5} \int_{s_{j_{0}+1}}^{s_{j_{0}+2}} f(v) d v
$$

and

$$
\int_{k_{f}-2}^{b} f(v) d v<c_{5} \int_{k_{f}-3}^{k_{f}-2} f(v) d v
$$

Hence

$$
\int_{x^{d}}^{b} f(v) d v<c_{6} \int_{s_{j_{0}+1}}^{k_{f}-2} f(v) d v
$$


and, therefore,

$$
E_{h}^{x} \zeta \geq c_{7} \int_{x^{d}}^{b} f(v) d v
$$

(ii) Next we will prove (ii) of Theorem 1.6.

First note that $k_{f}=\infty$. Recall the definitions of $j_{0}$ and the $T_{j}$ 's from part (i) of the proof. By Lemma 2.13 and the strong Markov property applied at $T_{j}$ 's, there exist non-negative (not necessarily independent) random variables $Z_{j}$ and i.i.d. non-negative random variables $Y_{j}$ such that

$$
\sum_{j=j_{0}+2}^{\infty}\left(T_{j}-T_{j-1}\right)
$$

has the same distribution as

$$
\sum_{j=j_{0}+2}^{\infty}\left(Z_{j}+f^{2}\left(s_{j-1}\right) Y_{j}\right) .
$$

For later use, note that, as in the proof of Lemma 2.13, we can write $Y_{j}=W_{j} Y_{j}^{\prime}$, where the $Y_{j}^{\prime}$ are independent of the $Z$ 's and $W^{\prime}$ 's, with some common mean $\mu$ and variance $\sigma^{2}$. Each $W_{j}$ takes values 0 or 1 , and $W_{j}=1$ with some common probability $p$, even if conditioned on the preceding $W$ 's and on $\left\{X_{t}, t \in\left[0, T_{j-1}\right]\right\}$. Thus the $W_{j}$ are i.i.d., though they may not be independent of the $Z_{j}$.

It is elementary to check that $\sum_{j=j_{0}+2}^{\infty} f^{2}\left(s_{j-1}\right)=\infty$ because $\int_{x^{d}}^{b} f(v) d v=\infty$. Hence,

$$
\sum_{j=j_{0}+2}^{\infty} E\left(f^{2}\left(s_{j-1}\right) Y_{j}\right)=\infty .
$$

Recalling that each $Y_{j}$ is non-negative, non-constant and bounded, the three-series theorem now easily implies a.s. that

$$
\sum_{j=j_{0}+2}^{\infty} f^{2}\left(s_{j-1}\right) Y_{j}=\infty .
$$

It follows that the sums in (2.31), and therefore in (2.30), must be infinite a.s.

(iii) We are going to prove the lower bound in (1.8).

Let $j_{0}$, the $Y_{j}$ 's, etc. be as in part (ii) of the proof. By adjusting the first and last $Z$, if necessary, we can guarantee that

$$
\begin{aligned}
\zeta & =\sum_{j=j_{0}+2}^{k_{f}-1}\left(Z_{j}+f^{2}\left(s_{j-1}\right) Y_{j}\right) \\
& =\sum_{j=j_{0}+2}^{k_{f}-1}\left(Z_{j}+f^{2}\left(s_{j-1}\right) \mu W_{j}\right)+\sum_{j=j_{0}+2}^{k_{f}-1}\left(f^{2}\left(s_{j-1}\right) W_{j}\left(Y_{j}^{\prime}-\mu\right)\right) .
\end{aligned}
$$


Therefore by independence,

$$
\begin{aligned}
\operatorname{Var}_{h}^{x} \zeta & =\operatorname{Var}_{h}^{x}\left(\sum_{j=j_{0}+2}^{k_{f}-1}\left(Z_{j}+f^{2}\left(s_{j-1}\right) \mu W_{j}\right)\right)+\sum_{j=j_{0}+2}^{k_{f}-1} E_{h}^{x}\left(\left(f^{2}\left(s_{j-1}\right) W_{j}\left(Y_{j}^{\prime}-\mu\right)\right)^{2}\right) \\
& \geq \sum_{j=j_{0}+2}^{k_{f}-1} E_{h}^{x}\left(\left(f^{2}\left(s_{j-1}\right) W_{j}\left(Y_{j}^{\prime}-\mu\right)\right)^{2}\right) \\
& \geq \sum_{j=j_{0}+2}^{k_{f}-1} f^{4}\left(s_{j-1}\right) p \sigma^{2} \geq c_{3} \int_{x^{d}}^{b} f^{3}(v) d v .
\end{aligned}
$$

(iv) We will now prove part (v) of Theorem 1.6.

We will again invoke the $Y_{j}$ 's and $Z_{j}$ 's of part (ii) of the proof. Suppose that $\int_{x^{d}}^{b} f^{3}(v) d v=\infty$. Then necessarily $b=\infty$. Let us assume that

$$
\limsup _{v \rightarrow \infty} f(v)<\infty .
$$

In order to simplify the notation, suppose that $x^{d}=s_{j_{0}}$.

First, let $w_{1}, w_{2}, \ldots$ be any sequence of 0's and 1's, such that

$$
\sum_{j>j_{0}} f^{4}\left(s_{j-1}\right) w_{j}=\infty
$$

Consider

$$
\widetilde{Y}_{k}=\sum_{j=j_{0}+1}^{k} f^{2}\left(s_{j-1}\right) w_{j}\left(Y_{j}^{\prime}-\mu\right) \quad \text { and } \quad \widehat{Y}_{k}=\widetilde{Y}_{k} /\left(\operatorname{Var} \widetilde{Y}_{k}\right)^{1 / 2} .
$$

Since the $Y_{j}^{\prime}$ s are uniformly bounded, the Lindeberg-Feller condition can be easily verified using (2.33) and it follows that the distributions of $\widehat{Y}_{k}$ converge to the standard normal distribution as $k \rightarrow \infty$. In fact it is simple to show, using (2.33) and the Berry-Eseen theorem, that for every $c_{1}<\infty$ and $c_{2}>0$ there exists a $c_{3}<\infty$ such that

$$
P\left(\widetilde{Y}_{k} \in\left(u, u+c_{1}\right)\right)<c_{2} / 2 \text { for every } u \in \mathbb{R}, \quad \text { if } \operatorname{Var} \widetilde{Y}_{k}>c_{3} .
$$

Since $\sum_{j>j_{0}} f^{4}\left(s_{j-1}\right) W_{j}=\infty$ almost surely, we can choose a $k_{0}<\infty$ such that

$$
P_{h}^{x}\left(\sum_{j=j_{0}+1}^{k} f^{4}\left(s_{j-1}\right) W_{j}>c_{3}\right)>1-c_{2} / 2
$$

for every $k \geq k_{0}$. Also, as in (2.32) we have that

$$
T\left(\Lambda_{s_{k}}\right)=\sum_{j=j_{0}+2}^{k}\left(Z_{j}+f^{2}\left(s_{j-1}\right) \mu W_{j}\right)+\sum_{j=j_{0}+2}^{k}\left(f^{2}\left(s_{j-1}\right) W_{j}\left(Y_{j}^{\prime}-\mu\right)\right) .
$$

Therefore, conditioning on the values of $W_{j}, j>j_{0}$ yields that

$$
P_{h}^{x}\left(T\left(\Lambda_{s_{k}}\right) \in\left(u, u+c_{1}\right)\right)<c_{2}
$$

for every $u \in \mathbb{R}$.

The case when (2.33) fails is not hard and is left to the reader.

(v) Next we prove the upper bound in (1.7). 
Suppose that $s_{n+1} \leq x^{d} \leq s_{n+2}$. Let $L$ be the last exit time from $\Lambda_{s_{n}}$. Under $P_{h}^{x}$, the process $\left\{X_{t}, t \in[0, L]\right\}$ is a conditioned Brownian motion in $D_{f}$ starting from $x$ and converging to a (random) point of $\Lambda_{s_{n}}$. Lemma 2.22 implies that $E_{h}^{x} L<c_{1} f^{2}\left(s_{n}\right)$ and this in turn implies that

$$
E_{h}^{x} L<c_{2} \int_{x^{d}}^{s_{n+3}} f(v) d v
$$

For every $\varepsilon>0$, the process $\left\{X_{t+L+\varepsilon}, t \geq 0\right\}$ under $P_{h}^{x}$ is an $h$-process in the domain $D_{g}$ where $g(s)=f(s) \mathbf{1}_{\left(s_{n}, \infty\right)}(s)$. This and (2.34) show that (1.7) will follow once we prove that

$$
E_{h}^{x} \zeta<c_{3} \int_{a}^{b} f(v) d v
$$

Let $M_{k}=\left\{y \in D_{f}: s_{k-1}<y^{d}<s_{k+1}\right\}$ and consider an $h_{0}$-process in $M_{k}$ for some positive harmonic function $h_{0}$ in $M_{k}$. A variation of Lemma 2.6 shows that

$$
E_{h_{0}}^{y} \zeta<c_{4}
$$

for all $y \in M_{k}$, provided $f\left(s_{k}\right)=1$. By scaling,

$$
E_{h_{0}}^{y} \zeta<c_{4} f^{2}\left(s_{k}\right)
$$

for any value of $f\left(s_{k}\right)$.

Recall the stopping times $S_{j}^{k}$ and $T_{j}^{k}$ from Lemma 2.16 and let $F_{j}^{k} \stackrel{\text { df }}{=}\left\{T_{j}^{k}<\infty\right\}$. Let $T_{0}$ be the hitting time of $\bigcup_{k} \Lambda_{s_{k}}$. We have

$$
\zeta=T_{0}+\sum_{j, k}\left(S_{j}^{k}-T_{j}^{k}\right) \mathbf{1}_{F_{j}^{k}} .
$$

Given $T_{j}^{k}<\infty$, the process $\left\{X_{t}, t \in\left[T_{j}^{k}, S_{j}^{k}\right]\right\}$ is a conditioned Brownian motion in $M_{k}$ and, therefore,

$$
E_{h}^{x}\left[\left(S_{j}^{k}-T_{j}^{k}\right) \mid F_{j}^{k}\right]<c_{4} f^{2}\left(s_{j}\right)
$$

By Lemma 2.16,

$$
\sum_{k} E_{h}^{x}\left(S_{j}^{k}-T_{j}^{k}\right) \mathbf{1}_{F_{j}^{k}}<c_{5} f^{2}\left(s_{j}\right) .
$$

Recall that $s_{n+1} \leq x^{d} \leq s_{n+2}$. Hence $E_{h}^{x} T_{0}<c_{4} f^{2}\left(s_{n}\right)$. This and (2.37)-(2.38) yield

$$
E_{h}^{x} \zeta \leq c_{6} \sum_{j} f^{2}\left(s_{j}\right)
$$

It is easy to check that the last quantity is bounded by $c_{7} \int_{a}^{b} f(v) d v$.

(vi) We will now prove the upper bound for the variance in (1.8). Recall $M_{k}$ and the use of an $h_{0}$-process in $M_{k}$ from part (v) of the proof. The Chebyshev inequality and (2.35) show that $P_{h_{0}}^{x}\left(\zeta>c_{1}\right)<c_{2}$ for some $c_{1}<\infty, c_{2}<1$ and all $x \in M_{k}$ provided $f\left(s_{k}\right)=1$. By the Markov property applied repeatedly at the multiples of $c_{1}, P_{h_{0}}^{x}\left(\zeta>j c_{1}\right)<c_{2}^{j}$. Hence $E_{h_{0}}^{x} \zeta^{2}<c_{3}$ in the case $f\left(s_{k}\right)=1$ and, by scaling,

$$
E_{h_{0}}^{x} \zeta^{2}<c_{3} f^{4}\left(s_{k}\right)
$$

for any value of $f\left(s_{k}\right)$, all $x \in M_{k}$ and all harmonic functions $h_{0}$ in $M_{k}$. 
Let $S_{j}^{k}$ and $T_{j}^{k}$ be as in Lemma 2.16. Let $F_{j}^{k} \stackrel{\text { df }}{=}\left\{T_{j}^{k}<\infty\right\}$. Given $F_{j}^{k}$, the process $\left\{X_{t}, t \in\left[T_{j}^{k}, S_{j}^{k}\right]\right\}$ is a conditioned Brownian motion in $M_{k}$ and this implies in view of (2.36) and (2.39), that

$$
\begin{gathered}
E_{h}^{x}\left[\left(S_{j}^{k}-T_{j}^{k}\right) \mid F_{j}^{k}\right]<c_{4} f^{2}\left(s_{j}\right) \quad \text { and } \\
E_{h}^{x}\left[\left(S_{j}^{k}-T_{j}^{k}\right)^{2} \mid F_{j}^{k}\right]<c_{3} f^{4}\left(s_{j}\right) .
\end{gathered}
$$

Let $\Theta_{j}^{k} \stackrel{\text { df }}{=}\left(S_{j}^{k}-T_{j}^{k}\right) \mathbf{1}_{F_{j}^{k}}$. Define $q$ by the condition that $s_{q-1}<x^{d} \leq s_{q}$, and recall from Lemma 2.16 that

$$
P_{h}^{x}\left(F_{j}^{k}\right) \leq \begin{cases}c_{5} c_{6}^{k+q-j}, & j<q, \\ c_{5} c_{6}^{k}, & j \geq q,\end{cases}
$$

where $c_{6}<1$. This and (2.40) imply that

$$
E_{h}^{x}\left[\Theta_{j}^{k}\right] \leq \begin{cases}c_{4} c_{5} c_{6}^{k+q-j} f^{2}\left(s_{j}\right), & j<q, \\ c_{4} c_{5} c_{6}^{k} f^{2}\left(s_{j}\right), & j \geq q,\end{cases}
$$

and

$$
E_{h}^{x}\left[\left(\Theta_{j}^{k}\right)^{2}\right] \leq \begin{cases}c_{3} c_{5} c_{6}^{k+q-j} f^{4}\left(s_{j}\right), & j<q, \\ c_{3} c_{5} c_{6}^{k} f^{4}\left(s_{j}\right), & j \geq q,\end{cases}
$$

Now assume that $j<n$, and let

$$
A=\left\{T_{j}^{k}<T_{n}^{1}\right\}, \quad B=\left\{T_{n}^{1}<T_{j}^{k}\right\}, \quad B_{i}=\left\{T_{j}^{i-1}<T_{n}^{1}<T_{j}^{i}\right\}
$$

where $T_{j}^{0}$ is taken to be 0 . Then

$$
\begin{aligned}
\operatorname{Cov}_{h}^{x}\left(\Theta_{j}^{k}, \Theta_{n}^{m}\right)= & E_{h}^{x}\left(\left(\Theta_{j}^{k}-E_{h}^{x} \Theta_{j}^{k}\right)\left(\Theta_{n}^{m}-E_{h}^{x} \Theta_{n}^{m}\right)\right) \\
= & E_{h}^{x}\left(\left(\Theta_{j}^{k}-E_{h}^{x} \Theta_{j}^{k}\right)\left(\Theta_{n}^{m}-E_{h}^{x} \Theta_{n}^{m}\right) \mathbf{1}_{A}\right) \\
& \quad+E_{h}^{x}\left(\left(\Theta_{j}^{k}-E_{h}^{x} \Theta_{j}^{k}\right)\left(\Theta_{n}^{m}-E_{h}^{x} \Theta_{n}^{m}\right) \mathbf{1}_{B}\right) \\
\stackrel{\mathrm{df}}{=} & I+I I .
\end{aligned}
$$

Consider term $I$ of (2.44). If $q>n$, then $I=0$ automatically. So suppose that $q \leq j$. By Corollary 2.28 and the strong Markov property at $T_{n}^{1}$,

$$
\left|E_{h}^{y} \Theta_{n}^{m}-E_{h}^{x} \Theta_{n}^{m}\right| \leq c_{7} c_{8}^{n-j} E_{h}^{x} \Theta_{n}^{m}
$$

for any $y \in D_{j}$, where $c_{8}<1$. In particular,

$$
\left|E_{h}^{x}\left(\Theta_{n}^{m} \mid \mathcal{F}_{S_{j}^{k}}\right)-E_{h}^{x} \Theta_{n}^{m}\right| \leq c_{7} c_{8}^{n-j} E_{h}^{x}\left(\Theta_{n}^{m}\right)
$$

on $A$. Thus, by (2.42),

$$
\begin{aligned}
I & =E_{h}^{x}\left[\left(\Theta_{j}^{k}-E_{h}^{x} \Theta_{j}^{k}\right) \mathbf{1}_{A} E_{h}^{x}\left(\Theta_{n}^{m}-E_{h}^{x}\left(\Theta_{n}^{m}\right) \mid \mathcal{F}_{S_{j}^{k}}\right)\right] \\
& \leq E_{h}^{x}\left[\left|\Theta_{j}^{k}-E_{h}^{x} \Theta_{j}^{k}\right| \cdot \mathbf{1}_{A} \cdot\left|E_{h}^{x}\left(\Theta_{n}^{m} \mid \mathcal{F}_{S_{j}^{k}}\right)-E_{h}^{x} \Theta_{n}^{m}\right|\right] \\
& \leq 2 c_{7} c_{8}^{n-j} E_{h}^{x}\left(\Theta_{n}^{m}\right) E_{h}^{x}\left(\Theta_{j}^{k}\right) \leq c_{9} c_{8}^{n-j} c_{6}^{k+m} f^{2}\left(s_{j}\right) f^{2}\left(s_{n}\right) .
\end{aligned}
$$

If, on the other hand, we have $j<q \leq n$, then by a similar argument,

$$
\left|E_{h}^{x}\left(\Theta_{n}^{m} \mid \mathcal{F}_{S_{j}^{k}}\right)-E_{h}^{x} \Theta_{n}^{m}\right| \leq c_{7} c_{8}^{n-q} E_{h}^{x}\left(\Theta_{n}^{m}\right)
$$

on $A$, and

$$
I \leq 2 c_{7} c_{8}^{n-q} E_{h}^{x}\left(\Theta_{n}^{m}\right) E_{h}^{x}\left(\Theta_{j}^{k}\right) \leq c_{9} c_{8}^{n-q} c_{6}^{k+m+q-j} f^{2}\left(s_{j}\right) f^{2}\left(s_{n}\right) .
$$


Taking $c_{11}=\max \left(c_{8}, c_{6}\right)$, it follows that

$$
I \leq c_{9} c_{11}^{n-j} c_{6}^{k+m} f^{2}\left(s_{j}\right) f^{2}\left(s_{n}\right),
$$

regardless of the value of $q$.

Consider now the term II of (2.44). By (2.43), and by Lemma 2.16 again,

$$
\begin{aligned}
E_{h}^{x}\left(\left(\Theta_{j}^{k}\right)^{2} \mathbf{1}_{B}\right) & =\sum_{i=1}^{k} E_{h}^{x}\left(\left(\Theta_{j}^{k}\right)^{2} \mathbf{1}_{B_{i}}\right) \\
& =\sum_{i=1}^{k} E_{h}^{x}\left(E_{h}^{x}\left(\left(\Theta_{j}^{k}\right)^{2} \mathbf{1}_{B_{i}} \mid \mathcal{F}_{S_{n}^{1}}\right)\right) \\
& \leq \sum_{i=1}^{k} f^{4}\left(s_{j}\right) c_{0} c_{3} c_{6}^{n-j+k-i+1} P_{h}^{x}\left(B_{i}\right) \\
& \leq \sum_{i=1}^{k} f^{4}\left(s_{j}\right) c_{0} c_{3} c_{6}^{n-j+k-i+1} P_{h}^{x}\left(F_{j}^{i-1}\right) \\
& \leq k c_{0}^{2} c_{3} c_{6}^{n-j+k} f^{4}\left(s_{j}\right) \leq c_{12} c_{13}^{n-j+k} f^{4}\left(s_{j}\right),
\end{aligned}
$$

where $c_{13}<1$. As a result,

$$
\begin{aligned}
I I & \leq\left(E_{h}^{x}\left(\left(\Theta_{j}^{k}\right)^{2} \mathbf{1}_{B}\right)\right)^{1 / 2}\left(E_{h}^{x}\left(\left(\Theta_{n}^{m}\right)^{2}\right)\right)^{1 / 2} \\
& \leq f^{2}\left(s_{j}\right) f^{2}\left(s_{n}\right)\left(c_{12} c_{13}^{n-j+k} c_{0} c_{3} c_{6}^{m}\right)^{1 / 2} \\
& \leq c_{14} c_{15}^{k+m+n-j} f^{2}\left(s_{j}\right) f^{2}\left(s_{n}\right),
\end{aligned}
$$

where $c_{14}<1$. Combining this with $(2.44)$ and (2.45), it follows that

$$
\operatorname{Cov}_{h}^{x}\left(\Theta_{j}^{k}, \Theta_{n}^{m}\right) \leq c_{16} c_{17}^{k+m+|n-j|} f^{2}\left(s_{j}\right) f^{2}\left(s_{n}\right),
$$

for $j<n$, where $c_{17}<1$. By symmetry, the same is true for $j>n$, and the inequality is even simpler to prove if $j=n((2.45)$ is no longer needed). Thus, (2.46) holds for every $j, k, m, n$.

If $\int_{a}^{b} f^{3}(v) d v=\infty$, then the upper bound in (1.8) is trivial. Assume therefore that $\int_{a}^{b} f^{3}(v) d v<\infty$. Then for each $\varepsilon>0$ there are only finitely many $j$ such that $f\left(s_{j}\right)>\varepsilon$. Hence we may choose an ordering $\left\{j_{i}\right\}_{i \geq 1}$ of the set $\left\{k: a<s_{k}<b\right\}$ which satisfies $f\left(s_{j_{i+1}}\right) \leq f\left(s_{j_{i}}\right)$ for all $i$. By $(2.46)$

$$
\begin{aligned}
\operatorname{Var}_{h}^{x} \zeta & =\operatorname{Var}_{h}^{x}\left(\sum_{j, k} \Theta_{j}^{k}\right)=\sum_{j, k, n, m} \operatorname{Cov}_{h}^{x}\left(\Theta_{j}^{k}, \Theta_{n}^{m}\right) \\
& \leq 2 \sum_{i} \sum_{n \geq i} \sum_{k} \sum_{m} \operatorname{Cov}_{h}^{x}\left(\Theta_{j_{i}}^{k}, \Theta_{j_{n}}^{m}\right) \\
& \leq 2 \sum_{i} \sum_{n \geq i} \sum_{k} \sum_{m} c_{13} c_{12}^{k+m+\left|j_{n}-j_{i}\right|} f^{2}\left(s_{j_{i}}\right) f^{2}\left(s_{j_{n}}\right) \\
& \leq \sum_{i} \sum_{n \geq i} c_{14} c_{12}^{\left|j_{n}-j_{i}\right|} f^{4}\left(s_{j_{i}}\right) \\
& \leq \sum_{j} c_{15} f^{4}\left(s_{j}\right) \leq c_{16} \int_{a}^{b} f^{3}(v) d v .
\end{aligned}
$$

(vii) Next we will prove part (iv) of Theorem 1.6. 
Fix some $x \in D_{f}$ and suppose for convenience that $x^{d}=s_{q}$ for some $q$. Recall $S_{j}^{k}, T_{j}^{k}, F_{j}^{k}$ and $\Theta_{j}^{k}$ from part (v) of the proof. With slightly more work, the argument for (2.46) can be seen to yield the following improved estimate:

$$
\operatorname{Cov}_{h}^{x}\left(\Theta_{j}^{k}, \Theta_{n}^{m}\right) \leq \begin{cases}c_{1} c_{2}^{k+m+|n-j|} f^{2}\left(s_{j}\right) f^{2}\left(s_{n}\right), & j, n \geq q, \\ c_{1} c_{2}^{k+m+|n-j|} c_{3}^{q-j} f^{2}\left(s_{j}\right) f^{2}\left(s_{n}\right), & j<q \leq n, \\ c_{1} c_{2}^{k+m+|n-j|} c_{3}^{q-j} c_{3}^{q-n} f^{2}\left(s_{j}\right) f^{2}\left(s_{n}\right), & j, n<q,\end{cases}
$$

where $c_{2}, c_{3}<1$.

Now we assume that the Lipschitz constant of $f$ is so small that for each $j$,

$$
\frac{f^{2}\left(s_{j-1}\right)}{f^{2}\left(s_{j}\right)}<\frac{c_{3}^{-1}+1}{2}
$$

Therefore

$$
\operatorname{Cov}_{h}^{x}\left(\Theta_{j}^{k}, \Theta_{n}^{m}\right) \leq \begin{cases}c_{1} c_{2}^{k+m+|n-j|} f^{2}\left(s_{j}\right) f^{2}\left(s_{n}\right), & j, n \geq q, \\ c_{1} c_{2}^{k+m+|n-j|} c_{4}^{q-j} f^{2}\left(s_{q}\right) f^{2}\left(s_{n}\right), & j<q \leq n, \\ c_{1} c_{2}^{k+m+|n-j|} c_{4}^{q-j} c_{4}^{q-n} f^{4}\left(s_{q}\right), & j, n<q,\end{cases}
$$

for some $c_{4}<1$.

If $\int_{x^{d}}^{b} f^{3}(v) d v=\infty$, then (1.9) obviously holds. Assume that $\int_{x^{d}}^{b} f^{3}(v) d v<\infty$. Then we may choose an ordering $\left\{j_{i}\right\}_{i \geq 1}$ of the set $\left\{k: x^{d} \leq s_{k}<b\right\}$ which satisfies $f\left(s_{j_{i+1}}\right) \leq f\left(s_{j_{i}}\right)$ for all $i$. Let $j_{i_{0}}=q$. Then in view of (2.48),

$$
\begin{aligned}
\operatorname{Var}_{h}^{x} \zeta= & \operatorname{Var}_{h}^{x}\left(\sum_{j, k} \Theta_{j}^{k}\right)=\sum_{j, k, n, m} \operatorname{Cov}_{h}^{x}\left(\Theta_{j}^{k}, \Theta_{n}^{m}\right) \\
\leq 2 & \sum_{i} \sum_{n \geq i} \sum_{k} \sum_{m} \operatorname{Cov}_{h}^{x}\left(\Theta_{j_{i}}^{k}, \Theta_{j_{n}}^{m}\right) \\
& +2 \sum_{j \leq n<q} \sum_{k} \sum_{m} \operatorname{Cov}_{h}^{x}\left(\Theta_{j}^{k}, \Theta_{n}^{m}\right) \\
& +2 \sum_{j<q} \sum_{i \geq i_{0}} \sum_{k} \sum_{m} \operatorname{Cov}_{h}^{x}\left(\Theta_{j}^{k}, \Theta_{j_{i}}^{m}\right) \\
& +2 \sum_{j<q} \sum_{i<i_{0}} \sum_{k} \sum_{m} \operatorname{Cov}_{h}^{x}\left(\Theta_{j}^{k}, \Theta_{j_{i}}^{m}\right) \\
\leq 2 & \sum_{i} \sum_{n \geq i} \sum_{k} \sum_{m} c_{1} c_{2}^{k+m+\left|j_{n}-j_{i}\right|} f^{2}\left(s_{j_{i}}\right) f^{2}\left(s_{j_{n}}\right) \\
& +2 \sum_{j \leq n<q} \sum_{k} \sum_{m} c_{1} c_{2}^{k+m+|n-j|} c_{4}^{q-j} c_{4}^{q-n} f^{4}\left(s_{q}\right) \\
& +2 \sum_{j<q} \sum_{i \geq i_{0}} \sum_{k} \sum_{m} c_{1} c_{2}^{k+m+\left|j_{i}-j\right|} c_{4}^{q-j} f^{2}\left(s_{q}\right) f^{2}\left(s_{j_{i}}\right) \\
& +2 \sum_{j<q} \sum_{i<i_{0}} \sum_{k} \sum_{m} c_{1} c_{2}^{k+m+\left|j_{i}-j\right|} c_{4}^{q-j} f^{2}\left(s_{q}\right) f^{2}\left(s_{j_{i}}\right) \\
\leq & \sum_{i} c_{5} f^{4}\left(s_{j_{i}}\right)+c_{6} f^{4}\left(s_{q}\right)+c_{7} f^{4}\left(s_{q}\right)+\sum_{i} c_{8} f^{4}\left(s_{j_{i}}\right) \\
\leq & c_{9} \sum_{j \geq q} f^{4}\left(s_{j}\right) \leq c_{10} \int_{x^{d}}^{b} f^{3}(v) d v . \quad \square
\end{aligned}
$$


Because they use similar arguments to those just given, we include the following two subsidiary results in this section.

(2.49) Corollary. Suppose that $D_{f}$ and $h$ are as in Theorem 1.6. Assume that $\int_{a}^{b} f^{3}(v) d v<\infty$. Then

$$
\lim _{x^{d} \rightarrow \infty} \sup \left\{\operatorname{Var}_{h}^{x} T\left(\Lambda_{u}\right): u>x^{d}\right\}=0 .
$$

Proof. Recall the notation from the proof of Theorem 1.6. As in the proof of (2.47), for every $x$ and for every $u=s_{i}$,

$$
\operatorname{Var}_{h}^{x} T\left(\Lambda_{u}\right)=\sum_{j, k, n, m} \operatorname{Cov}_{h}^{x}\left(\Theta_{j}^{k} \mathbf{1}_{\left\{T_{j}^{k}<T\left(\Lambda_{u}\right)\right\}}, \Theta_{n}^{m} \mathbf{1}_{\left\{T_{n}^{m}<T\left(\Lambda_{u}\right)\right\}}\right) .
$$

An examination of the proof of (2.47) shows that the terms of this sum are bounded by the terms of an absolutely convergent series, uniformly in $x$ and in $u=s_{i}$. With a little more work, it is easy to see that this domination holds for $u \in(a, b)$ as well. For fixed $j, k, m$ and $n$,

$$
\operatorname{Cov}_{h}^{x}\left(\Theta_{j}^{k} \mathbf{1}_{\left\{T_{j}^{k}<T\left(\Lambda_{u}\right)\right\}}, \Theta_{n}^{m} \mathbf{1}_{\left\{T_{n}^{m}<T\left(\Lambda_{u}\right)\right\}}\right) \rightarrow 0
$$

as $x^{d} \rightarrow \infty$, uniformly in $u$, because of (2.41). This easily implies (2.50).

(2.51) Lemma. Assume that $D_{f}$ and $h$ are as in Theorem 1.6. Set

$$
f_{*}(v) \stackrel{\text { df }}{=} \sup _{u \geq v} f(u) .
$$

There exists a $c_{1}<\infty$ such that for all $u$ and all $x_{1}, x_{2} \in D_{f}$ with $x_{1}^{d}=x_{2}^{d}<u$ we have

$$
\left|E_{h}^{x_{1}} T\left(\Lambda_{u}\right)-E_{h}^{x_{2}} T\left(\Lambda_{u}\right)\right| \leq c_{1} f_{*}^{2}\left(x_{1}^{d}\right) .
$$

Proof. We will use an argument from part (v) of the proof of Theorem 1.6. For simplicity, assume that $u=s_{m}$ for some $m$. Suppose that $s_{n+1} \leq x_{1}^{d} \leq s_{n+2}$ and let $L$ be the last exit from $\Lambda_{s_{n}}$. It has been proved that

$$
E_{h}^{x_{k}} L<c_{2} f^{2}\left(s_{n}\right)
$$

for $k=1,2$ (see the paragraph preceding (2.34)). Recall the definitions of $T_{0}, S_{j}^{k}, T_{j}^{k}$ and $F_{j}^{k}$ from the same proof, and set

$$
G_{j}^{k} \stackrel{\text { df }}{=} F_{j}^{k} \cap\left\{T_{j}^{k}<T\left(\Lambda_{u}\right)\right\} .
$$

We have

$$
E_{h}^{x_{k}} T_{0}<c_{3} f^{2}\left(s_{n+1}\right)
$$

by an argument analogous to that proving (2.36).

Let $g_{x_{i}}^{j}(y) d y=P_{h}^{x_{i}}\left(X\left(T_{j}^{1}\right) \in d y\right)$. By Corollary 2.28 it follows that for $j \geq n$,

$$
\begin{aligned}
\mid E_{h}^{x_{1}}\left[\left(S_{j}^{k}\right.\right. & \left.\left.-T_{j}^{k}\right) \mathbf{1}_{G_{j}^{k}}\right]-E_{h}^{x_{2}}\left[\left(S_{j}^{k}-T_{j}^{k}\right) \mathbf{1}_{G_{j}^{k}}\right] \mid \\
& =\left|\int_{\Lambda_{j}} E_{h}^{y}\left[\left(S_{j}^{k}-T_{j}^{k}\right) \mathbf{1}_{G_{j}^{k}}\right]\left(P_{h}^{x_{1}}\left(X\left(T_{j}^{1}\right) \in d y\right)-P_{h}^{x_{2}}\left(X\left(T_{j}^{1}\right) \in d y\right)\right)\right| \\
& \leq \int_{\Lambda_{j}} E_{h}^{y}\left[\left(S_{j}^{k}-T_{j}^{k}\right) \mathbf{1}_{G_{j}^{k}}\right]\left|1-\frac{g_{x_{2}}^{j}(y)}{g_{x_{1}}^{j}(y)}\right| P_{h}^{x_{1}}\left(X\left(T_{j}^{1}\right) \in d y\right) \\
& \leq c_{4} c_{5}^{|n-j|} E_{h}^{x_{1}}\left[\left(S_{j}^{k}-T_{j}^{k}\right) \mathbf{1}_{G_{j}^{k}}\right],
\end{aligned}
$$


where $c_{5}<1$. (Strictly speaking, Corollary 2.28 applies when $j>n+2$, but the other cases follow similarly.) Now (2.38) implies that

$$
\begin{aligned}
\mid \sum_{k} E_{h}^{x_{1}}\left(S_{j}^{k}-T_{j}^{k}\right) & \mathbf{1}_{G_{j}^{k}}-\sum_{k} E_{h}^{x_{2}}\left(S_{j}^{k}-T_{j}^{k}\right) \mathbf{1}_{G_{j}^{k}} \mid \\
& \leq c_{4} c_{5}^{|n-j|} \sum_{k} E_{h}^{x_{1}}\left(S_{j}^{k}-T_{j}^{k}\right) \mathbf{1}_{G_{j}^{k}} \\
& \leq c_{4} c_{5}^{|n-j|} c_{6} f^{2}\left(s_{j}\right) .
\end{aligned}
$$

Since

$$
\sum_{j \geq n} \sum_{k \geq 1}\left(S_{j}^{k}-T_{j}^{k}\right) \mathbf{1}_{G_{j}^{k}} \leq T\left(\Lambda_{u}\right) \leq T_{0}+L+\sum_{j \geq n} \sum_{k \geq 1}\left(S_{j}^{k}-T_{j}^{k}\right) \mathbf{1}_{G_{j}^{k}},
$$

we obtain from (2.52)-(2.54) that

$$
\begin{aligned}
& \left|E_{h}^{x_{1}} T\left(\Lambda_{u}\right)-E_{h}^{x_{2}} T\left(\Lambda_{u}\right)\right| \\
& \quad \leq 2 c_{2} f^{2}\left(s_{n}\right)+2 c_{3} f^{2}\left(s_{n+1}\right)+\sum_{j \geq n} c_{4} c_{5}^{|n-j|} c_{6} f^{2}\left(s_{j}\right) \\
& \quad \leq 2 c_{2} f_{*}^{2}\left(s_{n}\right)+2 c_{3} f_{*}^{2}\left(s_{n}\right)+\sum_{j \geq n} c_{4} c_{5}^{|n-j|} c_{6} f_{*}^{2}\left(s_{n}\right) \leq c_{7} f_{*}^{2}\left(s_{n}\right) .
\end{aligned}
$$

\section{Disintegration OF HARMONIC FUnCTIONS}

The purpose of this section is to prove Theorem 1.3. Unless otherwise indicated, the notation and general hypotheses of Theorem 1.3 will be assumed throughout this section.

Fix some $x_{0} \in D_{f}$ and let $g(u) \stackrel{\text { df }}{=}-E_{h}^{x_{0}} T\left(\Lambda_{u}\right)$. Recall that $f_{*}(v)=\sup _{u \geq v} f(u)$. Note that in either case (a) or (b) of Theorem 1.3 (i), we have that $f(v) \rightarrow 0$ as $v \rightarrow \infty$.

(3.1) Lemma. Suppose that one of the assumptions (a) or (b) of Theorem 1.3 (i) is satisfied. Then

$$
\lim _{u \rightarrow \infty}\left(T\left(\Lambda_{u}\right)+g(u)\right) \quad \text { exists } P_{h}^{x_{0}} \text {-a.s. }
$$

Proof. Lemma 2.51 and Corollary 2.49 show that for $k \geq 1$, we can choose $u_{k}$ such that

$$
\left|E_{h}^{x_{1}} T\left(\Lambda_{u}\right)-E_{h}^{x_{2}} T\left(\Lambda_{u}\right)\right| \leq c_{1} f_{*}^{2}\left(u_{k}\right) \leq 1 / k^{2}
$$

for all $x_{1}, x_{2} \in D_{f}$ and $u$ with $u_{k} \leq x_{1}^{d}=x_{2}^{d}<u$. We may also assume that

$$
\operatorname{Var}_{h}^{x} T\left(\Lambda_{u}\right) \leq 1 / k^{6}
$$

for $x \in D_{f}$ and $u$ with $u_{k} \leq x^{d}<u$.

Suppose $u \in\left[u_{k}, u_{k+1}\right)$. Since

$$
T\left(\Lambda_{u_{k+1}}\right)=\left(T\left(\Lambda_{u_{k+1}}\right)-T\left(\Lambda_{u}\right)\right)+T\left(\Lambda_{u}\right)
$$

we have

$$
g\left(u_{k+1}\right)=-E_{h}^{x_{0}}\left(T\left(\Lambda_{u_{k+1}}\right)-T\left(\Lambda_{u}\right)\right)+g(u) .
$$

This, (3.2), and the strong Markov property applied at $T\left(\Lambda_{u}\right)$ imply that

$$
\left|E_{h}^{x} T\left(\Lambda_{u_{k+1}}\right)+\left(g\left(u_{k+1}\right)-g(u)\right)\right| \leq 1 / k^{2}
$$


for all $x \in D_{f}$ such that $x^{d}=u$. The Chebyshev inequality and (3.3) yield that

$$
P_{h}^{x}\left(\left|T\left(\Lambda_{u_{k+1}}\right)-E_{h}^{x} T\left(\Lambda_{u_{k+1}}\right)\right| \geq 1 / k^{2}\right) \leq k^{4} \operatorname{Var}_{h}^{x} T\left(\Lambda_{u_{k+1}}\right) \leq 1 / k^{2},
$$

if $x^{d}=u$. This and (3.4) give

$$
P_{h}^{x}\left(\left|T\left(\Lambda_{u_{k+1}}\right)+\left(g\left(u_{k+1}\right)-g(u)\right)\right| \geq 2 / k^{2}\right) \leq 1 / k^{2},
$$

for $x \in D_{f}$ such that $x^{d}=u$. By the strong Markov property applied at $T\left(\Lambda_{u}\right)$,

$$
P_{h}^{x}\left(\left|T\left(\Lambda_{u_{k+1}}\right)-T\left(\Lambda_{u}\right)+\left(g\left(u_{k+1}\right)-g(u)\right)\right| \geq 2 / k^{2}\right) \leq 1 / k^{2},
$$

for any $x \in D_{f}$ with $x^{d} \leq u$. In particular,

$$
P_{h}^{x}\left(\left|T\left(\Lambda_{u_{k+1}}\right)-T\left(\Lambda_{u_{k}}\right)+\left(g\left(u_{k+1}\right)-g\left(u_{k}\right)\right)\right| \geq 2 / k^{2}\right) \leq 1 / k^{2},
$$

if $x^{d} \leq u_{k}$.

Fix some $c_{2}>0$ and find $j_{0}$ so large that $\sum_{j \geq j_{0}} 2 / j^{2}<c_{2}$. Suppose that $k>j_{0}, x^{d} \leq u$, and recall that $u \in\left[u_{k}, u_{k+1}\right)$. Then (3.5)-(3.6) imply that with $P_{h}^{x}$-probability larger than $1-c_{2}$, the event

$$
\begin{aligned}
&\left\{\left|T\left(\Lambda_{u_{k+1}}\right)-T\left(\Lambda_{u}\right)+\left(g\left(u_{k+1}\right)-g(u)\right)\right| \leq 2 / k^{2}\right\} \\
& \cap \bigcap_{j \geq j_{0}}\left\{\left|T\left(\Lambda_{u_{j+1}}\right)-T\left(\Lambda_{u_{j}}\right)+\left(g\left(u_{j+1}\right)-g\left(u_{j}\right)\right)\right| \leq 2 / j^{2}\right\}
\end{aligned}
$$

occurs. Let

$$
A_{v} \stackrel{\mathrm{df}}{=}\left\{\left|\left(T\left(\Lambda_{u_{m}}\right)+g\left(u_{m}\right)\right)-\left(T\left(\Lambda_{v}\right)+g(v)\right)\right|<c_{2} \quad \forall u_{m} \geq v\right\} .
$$

If the event in (3.7) holds, then $A_{u}$ holds, because in such a case we have

$$
\begin{aligned}
\mid\left(T\left(\Lambda_{u_{m}}\right)+g\left(u_{m}\right)\right)- & \left(T\left(\Lambda_{u}\right)+g(u)\right) \mid \\
\leq & \left|\left(T\left(\Lambda_{u_{k+1}}\right)+g\left(u_{k+1}\right)\right)-\left(T\left(\Lambda_{u}\right)+g(u)\right)\right| \\
& \quad+\sum_{j=k+1}^{m-1}\left|\left(T\left(\Lambda_{u_{j+1}}\right)-T\left(\Lambda_{u_{j}}\right)\right)+\left(g\left(u_{j+1}\right)-g\left(u_{j}\right)\right)\right| \\
\leq & 2 / k^{2}+\sum_{j=k+1}^{m-1} 2 / j^{2}<c_{2} .
\end{aligned}
$$

Hence $P_{h}^{x}\left(A_{u}\right)>1-c_{2}$.

Let

$$
W=W(u) \stackrel{\mathrm{df}}{=} \inf \left\{v>u:\left|\left(T\left(\Lambda_{v}\right)+g(v)\right)-\left(T\left(\Lambda_{u}\right)+g(u)\right)\right| \geq 2 c_{2}\right\} .
$$

By the strong Markov property applied at $T\left(\Lambda_{W}\right)$ we have $P_{h}^{x_{0}}\left(A_{W} \mid W<\infty\right)>$ $1-c_{2}$. Since $A_{u} \cap\{W<\infty\} \cap A_{W}=\emptyset$, it follows that $P_{h}^{x_{0}}\left(A_{W} \cap\{W<\infty\}\right)<c_{2}$, and hence $P_{h}^{x_{0}}(W<\infty)<c_{2} /\left(1-c_{2}\right)$. This proves the lemma, since we may assume that $c_{2}>0$ is arbitrarily small by choosing $u$ sufficiently large.

We now make some general observations about parabolic Martin boundaries. Let $D$ be a domain. For $\phi$ a parabolic function on $\dot{D}$, and $v<0$, define

$$
\phi_{v}(x, t) \stackrel{\mathrm{df}}{=} \phi(x, t+v) .
$$

Then $\phi_{v}$ is also parabolic. Moreover, if $\phi$ is minimal, then $\phi_{v}$ is either minimal or $\phi_{v} \equiv 0$ (see 1.XV.17 in [Db]). 
(3.8) Lemma. Let $D$ be a domain. Let $\phi$ be parabolic on $\dot{D}$, and let $v<0$. Then the laws of $X$ under $P_{\phi_{v}}^{x, t}$ and $P_{\phi}^{x, t+v}$ are the same.

Proof. It suffices to show that $P_{\phi_{v}}^{x, t}(A)=P_{\phi}^{x, t+v}(A)$, for $A$ an event of the form $\left\{X\left(t_{1}\right) \in A_{1}, \ldots, X\left(t_{n}\right) \in A_{n}\right\}$, where $t_{1}<t_{2}<\cdots<t_{n}$. But

$$
\begin{aligned}
P_{\phi_{v}}^{x, t}(A) & =\frac{1}{\phi_{v}(x, t)} E^{x, t}\left[\mathbf{1}_{A} \phi_{v}\left(X_{t_{n}}, \tau_{t_{n}}\right)\right] \\
& =\frac{1}{\phi(x, t+v)} E^{x, t}\left[\mathbf{1}_{A} \phi\left(X_{t_{n}}, \tau_{t_{n}}+v\right)\right] \\
& =\frac{1}{\phi(x, t+v)} E^{x, t+v}\left[\mathbf{1}_{A} \phi\left(X_{t_{n}}, \tau_{t_{n}}\right)\right]=P_{\phi}^{x, t+v}(A) .
\end{aligned}
$$

Now, if $\left(y_{k}, t_{k}\right) \in \dot{D},\left(y_{k}, t_{k}\right) \rightarrow z \in \partial^{M} \dot{D}$, and each $t_{k}<v$, then

$$
\begin{aligned}
\dot{K}\left((x, t),\left(y_{k}, t_{k}-v\right)\right) & =\frac{p_{t-t_{k}+v}^{D}\left(x, y_{k}\right)}{p_{-t_{k}+v}^{D}\left(x_{0}, y_{k}\right)} \\
& =\frac{p_{t-t_{k}+v}^{D}\left(x, y_{k}\right)}{p_{-t_{k}}^{D}\left(x_{0}, y_{k}\right)} \cdot \frac{p_{-t_{k}}^{D}\left(x_{0}, y_{k}\right)}{p_{-t_{k}+v}^{D}\left(x_{0}, y_{k}\right)} \\
& \rightarrow \frac{\dot{K}((x, t+v), z)}{\dot{K}\left(\left(x_{0}, v\right), z\right)} .
\end{aligned}
$$

Thus, provided $\dot{K}\left(\left(x_{0}, v\right), z\right)>0$, it follows that $\left(y_{k}, t_{k}-v\right)$ converges in $\dot{D}^{M}$ to a point $\Phi_{v} z \in \partial^{M} \dot{D}$ with

$$
\dot{K}\left(\cdot, \Phi_{v} z\right)=\frac{\dot{K}_{v}(\cdot, z)}{\dot{K}_{v}\left(\left(x_{0}, 0\right), z\right)} .
$$

Of course, it may happen that $\Phi_{v} z=z$. Note also that

$$
\dot{K}\left(\left(x_{0}, 0\right), \Phi_{v} z\right)=1,
$$

so that $\Phi_{v} z$ is a minimal point (according to the definition given in Section 1), if and only if $\dot{K}_{v}(\cdot, z)$ is a minimal function.

It would simplify several future arguments, if the map $\Phi_{v}$ could be defined for $v>0$ as well. A natural way of doing this would be to set

$$
\phi_{v}(x, t) \stackrel{\mathrm{df}}{=} \begin{cases}\phi(x, t+v), & t+v \leq 0 \\ \int p_{t+v}^{D}(x, y) \phi(y, 0) d y, & t+v>0 .\end{cases}
$$

The obstacle to this approach is that in general, this integral need not converge.

The following result is well known. See, for example, Theorems $\mathrm{C}$ and $\mathrm{E}$ of Aronson ([Ar], 1968).

(3.10) Lemma. Let $D$ be a domain, and let $A \subset \dot{D}$ be compact.

(i) Let $\varepsilon>0$ and $M<\infty$. There exists a $\delta>0$ such that if $u$ is parabolic on $\dot{D}$ and $u \leq M$, then $\left|u(z)-u\left(z^{\prime}\right)\right|<\varepsilon$ whenever $z, z^{\prime} \in A$ and $\left|z-z^{\prime}\right|<\delta$.

(ii) Let $x \in D$. There exists an $M<\infty$ such that if $u$ is parabolic on $\dot{D}$, and $u(x, 0) \leq 1$, then $u \leq M$ on $A$. 
(3.11) Lemma. Let $D$ be a domain. Suppose that $\left(y_{k}, t_{k}\right) \in \dot{D}$ converge to some $z \in \partial^{M} \dot{D}$, and that $a_{k} \rightarrow 0$. Let $(x, t) \in \dot{D}$ (so that, in particular, $t<0$ ) and suppose that $\dot{K}((x, t), z)>0$. Then

$$
\frac{p_{t-t_{k}}^{D}\left(x, y_{k}\right)}{p_{t-t_{k}-a_{k}}^{D}\left(x, y_{k}\right)} \rightarrow 1
$$

as $k \rightarrow \infty$. Moreover,

$$
\left(y_{k}, t_{k}+a_{k}-t\right) \rightarrow \Phi_{t} z .
$$

Proof. If $\dot{K}((x, t), z)>0$, then the $\dot{K}\left((x, t),\left(y_{k}, t_{k}\right)\right)$ are bounded away from 0 . Since $\dot{K}\left(\left(x_{0}, 0\right),\left(y_{k}, t_{k}\right)\right)=1$, (ii) of Lemma 3.10 shows that the $\dot{K}\left(\cdot,\left(y_{k}, t_{k}\right)\right)$ are uniformly bounded on a suitable neighbourhood of $(x, t)$. Applying (i) of Lemma 3.10 on this neighbourhood shows that

$$
\frac{p_{t-t_{k}}^{D}\left(x, y_{k}\right)}{p_{t-t_{k}-a_{k}}^{D}\left(x, y_{k}\right)}=\frac{\dot{K}\left((x, t),\left(y_{k}, t_{k}\right)\right)}{\dot{K}\left(\left(x, t-a_{k}\right),\left(y_{k}, t_{k}\right)\right)} \rightarrow 1,
$$

as $k \rightarrow \infty$, showing (3.12).

To prove (3.13), we must show that

$$
\lim _{k \rightarrow \infty} \dot{K}\left((x, s),\left(y_{k}, t_{k}+a_{k}-t\right)\right)=\lim _{k \rightarrow \infty} \dot{K}\left((x, s),\left(y_{k}, t_{k}-t\right)\right)
$$

for every $(x, s) \in \dot{D}$. But as before,

$$
\begin{gathered}
\dot{K}\left((x, s),\left(y_{k}, t_{k}+a_{k}-t\right)\right)=\frac{p_{s+t-t_{k}-a_{k}}\left(x, y_{k}\right)}{p_{t-t_{k}-a_{k}}\left(x_{0}, y_{k}\right)} \\
=\frac{\dot{K}\left(\left(x, s+t-a_{k}\right),\left(y_{k}, t_{k}\right)\right)}{\dot{K}\left(\left(x_{0}, t-a_{k}\right),\left(y_{k}, t_{k}\right)\right)} \\
\rightarrow \frac{\dot{K}((x, s+t), z)}{\dot{K}\left(\left(x_{0}, t\right), z\right)}=\dot{K}\left((x, s), \Phi_{t} z\right) .
\end{gathered}
$$

(3.14) Lemma. Assume that $f(u) \rightarrow 0$ as $u \rightarrow \infty$. Let $\left(z_{k}, t_{k}\right) \in \dot{D}_{f}$ converge to $z \in \partial^{M} \dot{D}_{f}$, and suppose that $\dot{K}((x, t), z)>0$ for every $(x, t) \in \dot{D}_{f}$. If $y_{k} \in D_{f}$ and $z_{k}^{d}=y_{k}^{d}$ for each $k$, then for some $c_{1}<\infty$ and $c_{2}>0$, and for every $q<0$ and $(x, t) \in \dot{D}_{f}$,

$$
\begin{gathered}
\limsup _{k \rightarrow \infty} \dot{K}\left((x, t),\left(y_{k}, t_{k}-q\right)\right) \leq c_{1} \dot{K}\left((x, t), \Phi_{q} z\right), \\
\liminf _{k \rightarrow \infty} \dot{K}\left((x, t),\left(y_{k}, t_{k}-q\right)\right) \geq c_{2} \dot{K}\left((x, t), \Phi_{q} z\right) .
\end{gathered}
$$

Proof. Let $r_{0}>0$ be so small that for each $w \in \partial D_{f}$, the set $\partial D_{f} \cap B\left(w, r_{0} f\left(w^{d}\right)\right)$ is the graph of a Lipschitz function $F$, with Lipschitz constant $\lambda_{0}$ in some orthonormal coordinate system $C S_{w}$. Let the coordinates of $x$ in $C S_{w}$ be $\left(\hat{x}, x^{\prime}\right)$, so that

$$
D_{f} \cap B\left(w, r_{0} f\left(w^{d}\right)\right)=\left\{\left(\hat{x}, x^{\prime}\right): x^{\prime}>F(\hat{x})\right\} \cap B\left(w, r_{0} f\left(w^{d}\right)\right) .
$$

Let

$$
\begin{aligned}
\Psi_{r}(w, s) & =\left\{(x, t) \in \dot{D}_{f}:|x-w|<r,|s-t|<r^{2}\right\} \\
A_{r}(w) & =\left(\hat{w}, w^{\prime}+r\right) \quad \text { in } C S_{w} .
\end{aligned}
$$


We fix a suitable $\bar{s}<0$ and apply Theorem 1.6 of Fabes, Garofalo and Salsa ([FGS], 1986) to some $\Psi_{r / 8}(w, \bar{s})$, to see that if $x_{1}, x_{2} \in D_{f}, w \in \partial D_{f}, r<r_{0} f\left(w^{d}\right) / 2$, $s, s^{\prime}<\bar{s}$ and $y \in B(w, r / 8)$, then

$$
\begin{aligned}
\frac{p_{-s}^{D_{f}}\left(x_{2}, y\right)}{p_{-s^{\prime}}^{D_{f}}\left(x_{1}, y\right)} & =\frac{\dot{G}_{\dot{D}_{f}}\left((y, \bar{s}),\left(x_{2}, \bar{s}+s\right)\right)}{\dot{G}_{\dot{D}_{f}}\left((y, \bar{s}),\left(x_{1}, \bar{s}+s^{\prime}\right)\right)} \\
& \leq c_{1} \frac{\dot{G}_{\dot{D}_{f}}\left(\left(A_{r}(w), \bar{s}+2 r^{2}\right),\left(x_{2}, \bar{s}+s\right)\right)}{\dot{G}_{\dot{D}_{f}}\left(\left(A_{r}(w), \bar{s}-2 r^{2}\right),\left(x_{1}, \bar{s}+s^{\prime}\right)\right)} \\
& =c_{1} \frac{p_{-s+2 r^{2}}^{D_{f}}\left(x_{2}, A_{r}(w)\right)}{p_{-s^{\prime}-2 r^{2}}\left(x_{1}, A_{r}(w)\right)} .
\end{aligned}
$$

Note that, although Theorem 1.6 of [FGS] would in principle allow the above constant $c_{1}$ to depend on $f\left(w^{d}\right)$, in fact a scaling argument shows that it does not.

Fix $(x, t) \in \dot{D}_{f}$ and $q<0$. Let $M=\bigcup_{w \in \partial D_{f}} B\left(w, r_{0} f\left(w^{d}\right) / 32\right)$. If $y_{k} \in M$, choose $w$ so that $y_{k} \in B(w, r / 8)$, where $r=r_{0} f\left(w^{d}\right) / 4$. With this choice of $r$, set

$$
\bar{y}_{k}=A_{r}(w), \quad a_{k}=2 r^{2} .
$$

If $y_{k} \notin M$, set

$$
\bar{y}_{k}=y_{k}, \quad a_{k}=0 .
$$

The assumption that $\dot{K}((x, t), z)>0$ for every $(x, t) \in \dot{D}_{f}$ easily implies that $t_{k} \rightarrow-\infty$. By (3.17),

$$
\frac{p_{t+q-t_{k}}^{D_{f}}\left(x, y_{k}\right)}{p_{q-t_{k}}^{D_{f}}\left(x_{0}, y_{k}\right)} \leq c_{1} \frac{p_{t+q-t_{k}+a_{k}}^{D_{f}}\left(x, \bar{y}_{k}\right)}{p_{q-t_{k}-a_{k}}^{D_{f}}\left(x_{0}, \bar{y}_{k}\right)},
$$

for $k$ so large that $t_{k}-t-q<\bar{s}$.

Let $b_{k}=f^{2}\left(z_{k}^{d}\right)$. A precise version of the parabolic Harnack principle (see Theorem 0.2 of [FGS]) implies that for $k$ large and for every $v \in D_{f}$ with $\left|v^{d}-z_{k}^{d}\right|<$ $f\left(z_{k}^{d}\right)$ and $v \notin M$, we have

$$
\frac{p_{t+q-t_{k}+a_{k}}^{D_{f}}\left(x, \bar{y}_{k}\right)}{p_{q-t_{k}-a_{k}}^{D_{f}}\left(x_{0}, \bar{y}_{k}\right)} \leq c_{2} \frac{p_{t+q-t_{k}+a_{k}+b_{k}}^{D_{f}}(x, v)}{p_{q-t_{k}-a_{k}-b_{k}}^{D_{f}}\left(x_{0}, v\right)} .
$$

As above, take $\bar{z}_{k}$ equal to either $z_{k}$ (if $\left.z_{k} \notin M\right)$, or an $A_{r}(w)$ (if $z_{k} \in B(w, r / 8)$, where $\left.r=r_{0} f\left(w^{d}\right) / 4\right)$. Take $d_{k}$ equal to 0 or $2 r^{2}$ respectively. Therefore

$$
\frac{p_{t+q-t_{k}+a_{k}+b_{k}}^{D_{f}}\left(x, \bar{z}_{k}\right)}{p_{q-t_{k}-a_{k}-b_{k}}^{D_{f}}\left(x_{0}, \bar{z}_{k}\right)} \leq c_{1} \frac{p_{t+q-t_{k}+a_{k}+b_{k}+d_{k}}^{D_{f}}\left(x, z_{k}\right)}{p_{q-t_{k}-a_{k}-b_{k}-d_{k}}^{D_{f}}\left(x_{0}, z_{k}\right)},
$$

for $k$ large, as before. Since $q<0, a_{k} \rightarrow 0, b_{k} \rightarrow 0$, and $d_{k} \rightarrow 0$, it follows from (3.12) that

$$
\begin{aligned}
\lim _{k \rightarrow \infty} \frac{p_{t+q-t_{k}+a_{k}+b_{k}+d_{k}}^{D_{f}}\left(x, z_{k}\right)}{p_{q-t_{k}-a_{k}-b_{k}-d_{k}}^{D_{f}}\left(x_{0}, z_{k}\right)} & =\lim _{k \rightarrow \infty} \frac{p_{t+q-t_{k}}^{D_{f}}\left(x, z_{k}\right)}{p_{q-t_{k}}^{D_{f}}\left(x_{0}, z_{k}\right)} \\
& =\lim _{k \rightarrow \infty} \dot{K}\left((x, t),\left(z_{k}, t_{k}-q\right)\right)=\dot{K}\left((x, t), \Phi_{q} z\right) .
\end{aligned}
$$


Thus, taking $v=\bar{z}_{k}$, it follows from this and (3.18)-(3.20) that

$$
\limsup _{k \rightarrow \infty} \dot{K}\left((x, t),\left(y_{k}, t_{k}-q\right)\right)=\limsup _{k \rightarrow \infty} \frac{p_{t+q-t_{k}}^{D_{f}}\left(x, y_{k}\right)}{p_{q-t_{k}}^{D_{f}}\left(x_{0}, y_{k}\right)} \leq c_{3} \dot{K}\left((x, t), \Phi_{q} z\right)
$$

as well, proving (3.15). The argument for (3.16) is similar.

We may improve upon the conclusion of Lemma (3.14), by assuming that $z$ is minimal:

(3.21) Lemma. Assume that $f(u) \rightarrow 0$ as $u \rightarrow \infty$. Let $\left(z_{k}, t_{k}\right) \in \dot{D}_{f}$ converge to a minimal point $z \in \partial_{0}^{M} \dot{D}_{f}$, and suppose that $\dot{K}((x, t), z)>0$ for every $(x, t) \in \dot{D}_{f}$. If $y_{k} \in D_{f}$ satisfy $z_{k}^{d}=y_{k}^{d}$ for each $k$, and $q_{k} \rightarrow q<0$, then $\left(y_{k}, t_{k}-q_{k}\right) \rightarrow \Phi_{q} z$; that is,

$$
\lim _{k \rightarrow \infty} \dot{K}\left((x, t),\left(y_{k}, t_{k}-q_{k}\right)\right)=\dot{K}\left((x, t), \Phi_{q} z\right)
$$

for every $(x, t) \in \dot{D}_{f}$.

Proof. We first consider the limit of $\left(y_{k}, t_{k}-q\right)$. If $w$ is any limit point of this sequence, then by (3.15) we have that

$$
\dot{K}(\cdot, w) \leq c_{1} \dot{K}\left(\cdot, \Phi_{q} z\right) .
$$

By minimality of $z$ (and hence $\Phi_{q} z$ ), in fact

$$
\dot{K}(\cdot, w)=c \dot{K}\left(\cdot, \Phi_{q} z\right)
$$

for some $c<\infty$. By (3.16) we must have $c>0$, so $w \neq \dot{0}$.

Let $k_{i}$ be a subsequence along which $\left(y_{k_{i}}, t_{k_{i}}-q\right) \rightarrow w$. By passing to a further subsequence, if necessary, we may also ensure that $\left(y_{k_{i}}, t_{k_{i}}-q / 2\right)$ converges to some $w^{\prime} \neq \dot{0}$. Then $w=\Phi_{q / 2} w^{\prime}$, so by (3.9),

$$
\dot{K}\left(\left(x_{0}, 0\right), w\right)=1=\dot{K}\left(\left(x_{0}, 0\right), \Phi_{q} z\right) .
$$

Thus $c=1$, and so $w=z$. Since $\Phi_{q} z$ is the only limit point of $\left(y_{k}, t_{k}-q\right)$, it follows that the sequence itself converges to $\Phi_{q} z$.

Similarly, $\left(y_{k}, t_{k}-q / 2\right) \rightarrow \Phi_{q / 2} z$. Since $\Phi_{q} z=\Phi_{q / 2}\left(\Phi_{q / 2} z\right)$, we may set $a_{k}=q-$ $q_{k}$, and apply (3.13) (with $\left.t=q / 2\right)$, to obtain, in addition, that $\left(y_{k}, t_{k}-q_{k}\right) \rightarrow \Phi_{q} z$, as required.

Proof of Theorem 1.3. (i) Assume either (a) or (b) of (i) of the theorem, and recall that this implies that $f(u) \rightarrow 0$ as $u \rightarrow \infty$.

Let $\mathcal{H}_{s}$ denote the set of points $z$ of the minimal Martin boundary $\partial_{0}^{M} \dot{D}_{f}$, such that $g(u)-\tau\left(\Lambda_{u}\right) \rightarrow s, P_{z}^{x_{0}, 0}$-a.s. Set $\mathcal{H}=\bigcup_{s \in \mathbb{R}} \mathcal{H}_{s}$. Recall that if $\phi$ is a minimal parabolic function, then the tail $\sigma$-field of every $\phi$-transform of space-time Brownian motion is trivial. By Lemma 3.1, the random variable $\lim _{u \rightarrow \infty} g(u)-\tau\left(\Lambda_{u}\right)$ is well defined $P_{h}^{x_{0}}$-a.s. It is clearly measurable with respect to the tail $\sigma$-field of $\dot{X}_{t}$, and SO

$$
h(x)=\int_{\mathcal{H}} \dot{K}((x, t), z) \mu(d z),
$$

for every $(x, t) \in \dot{D}_{f}$, where $\mu$ is some measure concentrated on $\mathcal{H}$. In particular, it follows that $\mathcal{H}_{s}$ is nonempty for some $s \in \mathbb{R}$. We will work towards proving that, 
in fact,

every $\mathcal{H}_{s}$ consists of a single point,

namely the $z_{s}$ of $(\mathrm{A})$.

In fact, the conclusion of (B) will follow immediately from (3.22), since $\mathcal{H}_{s_{1}}$ and $\mathcal{H}_{s_{2}}$ are disjoint if $s_{1} \neq s_{2}$.

For $z \in \mathcal{H}_{s}$, we have that $g(u)-\tau\left(\Lambda_{u}\right) \rightarrow s, P_{z}^{x_{0}, 0}$-a.s. A standard argument now shows that the same is true, $P_{z}^{x, t}$-a.s., for every $(x, t) \in \dot{D}_{f}$. Thus (D) will also follow immediately, once (3.22) is proven.

(ii) It is a routine matter to prove that if

$$
P_{h}^{x_{0}, 0}\left(\lim _{u \rightarrow \infty}\left(g(u)-\tau\left(\Lambda_{u}\right)\right) \in\left(s_{1}, s_{2}\right)\right)>0,
$$

then for every $s_{3} \in \mathbb{R}$,

$$
P_{h}^{x_{0}, 0}\left(\lim _{u \rightarrow \infty}\left(g(u)-\tau\left(\Lambda_{u}\right)\right) \in\left(s_{1}+s_{3}, s_{2}+s_{3}\right)\right)>0 .
$$

Hence, (3.23) holds for all $-\infty<s_{1}<s_{2}<\infty$. Therefore

$$
\mu\left(\bigcup_{s \in\left(s_{1}, s_{2}\right)} \mathcal{H}_{s}\right)>0
$$

for every such $s_{1}, s_{2}$. This will establish (C). Moreover, it shows that

$$
\exists\left\{s_{k}\right\}_{k \geq 1} \text { such that } \lim _{k \rightarrow \infty} s_{k}=\infty \text { and for every } k, \mathcal{H}_{s_{k}} \neq \emptyset .
$$

If $\phi=\dot{K}(\cdot, z)$, where $z \in \mathcal{H}_{s}$, and $v<0$, then by Lemma 3.8,

$$
\begin{aligned}
1 & =P_{\phi}^{x, t+v}\left(g(u)-\tau\left(\Lambda_{u}\right) \rightarrow s\right)=P_{\phi}^{x, t+v}\left(g(u)+T\left(\Lambda_{u}\right)-t-v \rightarrow s\right) \\
& =P_{\phi_{v}}^{x, t}\left(g(u)+T\left(\Lambda_{u}\right)-t-v \rightarrow s\right)=P_{\phi_{v}}^{x, t}\left(g(u)-\tau\left(\Lambda_{u}\right) \rightarrow s+v\right) ;
\end{aligned}
$$

that is, the pole of $\phi_{v}$ belongs to $\mathcal{H}_{s+v}$. Thus, $\Phi_{v}$ maps $\mathcal{H}_{s}$ into $\mathcal{H}_{s+v}$. Appealing to (3.24), we conclude that $\mathcal{H}_{s}$ is nonempty, for every $s \in \mathbb{R}$.

(iii) Let $s \in \mathbb{R}$, and pick $z \in \mathcal{H}_{s}$. For any sequence $u_{k} \rightarrow \infty$, we may set $y_{k}=$ $X\left(T\left(\Lambda_{u_{k}}\right)\right)$, and $t_{k}=\tau\left(\Lambda_{u_{k}}\right)$. Because $\dot{X}\left(T\left(\Lambda_{u_{k}}\right)\right) \rightarrow z$ in the Martin topology, $P_{z}^{x, 0}$-a.s., it follows that we have constructed a sequence $\left(y_{k}, t_{k}\right) \rightarrow z$ as in part (A), with $s_{k} \stackrel{\text { df }}{=} g\left(y_{k}^{d}\right)-t_{k} \rightarrow s$.

Next we will show that $\mathcal{H}_{s}$ consists of a single point for each $s$. Suppose to the contrary that $z, \widetilde{z} \in \mathcal{H}_{s}$ for some $s$. It is easy to see that we must have $\Phi_{s^{\prime}-s} z \neq \Phi_{s^{\prime}-s} \widetilde{z}$ for some $s^{\prime}<s$. Fix any sequence $u_{k} \rightarrow \infty$. Consider any sequence $\left(y_{k}, t_{k}\right) \rightarrow z$, with $g\left(y_{k}^{d}\right)-t_{k} \rightarrow s$ and $y_{k}^{d}=u_{k}$, constructed as in the previous paragraph. Let $\left(\widetilde{y}_{k}, \widetilde{t}_{k}\right)$ be the analogous sequence with $\left(\widetilde{y}_{k}, \widetilde{t}_{k}\right) \rightarrow \widetilde{z}, \widetilde{y}_{k}^{d}=u_{k}$ and $g\left(\widetilde{y}_{k}^{d}\right)-\widetilde{t}_{k} \rightarrow s$. Note that $t_{k}-\widetilde{t}_{k} \rightarrow 0$ because $y_{k}^{d}=\widetilde{y}_{k}^{d}=u_{k}, g\left(y_{k}^{d}\right)-t_{k} \rightarrow s$, and $g\left(\widetilde{y}_{k}^{d}\right)-\widetilde{t}_{k} \rightarrow s$. Lemma 3.21 implies that $\left(y_{k}, t_{k}+s-s^{\prime}\right) \rightarrow \Phi_{s^{\prime}-s} z$. But it also implies that $\left(y_{k}, t_{k}+s-s^{\prime}\right) \rightarrow \Phi_{s^{\prime}-s} \widetilde{z}$, because $y_{k}^{d}=\widetilde{y}_{k}^{d}$ and $t_{k}-\widetilde{t}_{k} \rightarrow 0$. This contradicts the fact that $\Phi_{s^{\prime}-s} z \neq \Phi_{s^{\prime}-s} \widetilde{z}$ and so it proves our claim, and establishes (3.22).

Now let $r_{k} \rightarrow s$, and consider any sequence $x_{k}$ such that $x_{k}^{d} \rightarrow \infty$. Our goal is to show that $\left(x_{k}, g\left(x_{k}^{d}\right)-r_{k}\right) \rightarrow z$, where $z$ is the only element of $\mathcal{H}_{s}$. Set $u_{k}=x_{k}^{d}$, and this time choose $s^{\prime}>s$. Let $z^{\prime}$ be the element of $\mathcal{H}_{s^{\prime}}$. Note that $\Phi_{s-s^{\prime}} z^{\prime}=z$. By the argument of the first paragraph of (iii), we may choose $\left(y_{k}, t_{k}^{\prime}\right) \rightarrow z^{\prime}$ with 
$y_{k}^{d}=u_{k}=x_{k}^{d}$ and $g\left(y_{k}^{d}\right)-t_{k}^{\prime} \rightarrow s^{\prime}$. Since $t_{k}^{\prime}-\left[g\left(x_{k}^{d}\right)-r_{k}\right] \rightarrow-s^{\prime}+s$, we may apply Lemma 3.21 and obtain that

$$
\left(x_{k}, g\left(x_{k}^{d}\right)-r_{k}\right) \rightarrow \Phi_{s-s^{\prime}} z^{\prime}=z .
$$

This finishes the proof of (A). Thus, part (i) of Theorem 1.3 is proven.

(iv) Turning to part (ii) of Theorem 1.3 , suppose that $\int_{u}^{\infty} f^{3}(v) d v=\infty$ for all $u<\infty$. We also assume, since it simplifies the proof, that $f(u) \rightarrow 0$ as $u \rightarrow \infty$. At the end we will sketch out how to extend the argument to the general case, that $\lim \sup _{u \rightarrow \infty} f(u)<\infty$.

We use a coupling argument. Fix $x_{1}, x_{2} \in D_{f}$, and $s \leq 0$. Let $X_{1}$ and $X_{2}$ be independent processes, under a probability measure $P$, with the same distributions as $X$ under $P_{h}^{x_{1}, s}$ and $P_{h}^{x_{2}, s}$ respectively. Thus, $\dot{X}_{1}(t)=\left(X_{1}(t), \tau_{t}\right)$ and $\dot{X}_{2}(t)=$ $\left(X_{2}(t), \tau_{t}\right)$ are versions of $\dot{X}$, where $\tau(t)=s-t$. Define

$$
W=\inf \left\{t>0: X_{1}^{d}(t)=X_{2}^{d}(t)\right\}
$$

We will show that

$$
P(W<\infty)=1
$$

Write $T_{j}\left(\Lambda_{u}\right)$ for the hitting time of $\Lambda_{u}$ by $X_{j}$. We may assume, without loss of generality, that $x_{1}^{d} \leq x_{2}^{d}$. Set $u_{0}=x_{2}^{d}+f\left(x_{2}^{d}\right), Y_{j}=T_{j}\left(\Lambda_{u_{0}}\right)$ and $Z_{j}=$ $T_{j}\left(\Lambda_{u}\right)-T_{j}\left(\Lambda_{u_{0}}\right)$, where the value of $u$ will be chosen later. A standard application of the boundary Harnack principle (Lemma 2.2) shows that the Radon-Nikodym derivative of the hitting distributions of $\Lambda_{u_{0}}$ under $P_{h}^{y_{1}}$ and $P_{h}^{y_{2}}$ is bounded below by $c_{1}>0$ for all $y_{1}, y_{2} \in \Lambda_{x_{2}^{d}}$.

Let $c_{2}$ be so large that

$$
P\left(Y_{1}-Y_{2} \geq c_{2}\right)<c_{1} / 16
$$

Use Theorem 1.6 (v) to find $u$ so large that for every $v \in \mathbb{R}$ we have

$$
P\left(Z_{2} \in\left(v, v+c_{2}\right)\right)<c_{1} / 8 \text {. }
$$

Let $v_{1}$ be the median of $Z_{1}$, in other words,

$$
P\left(Z_{1} \leq v_{1}\right) \geq 1 / 2, \quad P\left(Z_{1} \geq v_{1}\right) \geq 1 / 2 .
$$

By applying the strong Markov property at $T\left(\Lambda_{u_{0}}\right)$, and by our choice of $c_{1}$, we have $P\left(Z_{2} \geq v_{1}\right) \geq c_{1} / 2$. Now we use (3.27) to obtain that $P\left(Z_{2} \geq v_{1}+c_{2}\right) \geq 3 c_{1} / 8$. This, (3.28) and the independence of $Z_{1}$ and $Z_{2}$ show that

$$
P\left(Z_{2}-Z_{1} \geq c_{2}\right) \geq P\left(Z_{1} \leq v_{1}, Z_{2} \geq v_{1}+c_{2}\right) \geq 3 c_{1} / 16 .
$$

Inequality (3.26) now implies that

$$
\begin{aligned}
P\left(T_{1}\left(\Lambda_{u}\right)<T_{2}\left(\Lambda_{u}\right)\right) & =P\left(Y_{1}+Z_{1}<Y_{2}+Z_{2}\right) \\
& \geq P\left(Y_{1}-Y_{2}<c_{2} \leq Z_{2}-Z_{1}\right) \\
& \geq P\left(Z_{2}-Z_{1} \geq c_{2}\right)-P\left(Y_{1}-Y_{2} \geq c_{2}\right) \geq c_{1} / 8 .
\end{aligned}
$$

Let $V_{j}^{0}=x_{j}, \tau^{0}=s, T^{1}=\max \left(T_{1}\left(\Lambda_{u}\right), T_{2}\left(\Lambda_{u}\right)\right), \tau^{1}=\tau\left(T^{1}\right), V_{j}^{1}=X\left(T_{j}\left(\Lambda_{u}\right)\right)$, $U^{1}=u$. Repeat the above argument, starting from $\left(V_{j}^{1}, \tau^{1}\right)$ in place of $\left(V_{j}^{0}, \tau^{0}\right)$, and ensuring that $U^{2}$ is chosen so large that each $T_{j}\left(\Lambda_{U^{2}}\right)>T^{1}$. Then continue this procedure inductively, to obtain sequences of random variables $V_{j}^{k}, T^{k}, \tau^{k}$, and $U^{k}$. By the strong Markov property, (3.29) becomes

$$
P\left(T_{1}\left(\Lambda_{U^{k+1}}\right)<T_{2}\left(\Lambda_{U^{k+1}}\right) \mid \mathcal{F}_{T^{k}}\right) \geq c_{1} / 8,
$$


where $\mathcal{F}_{t}$ is the filtration of $\left(X_{1}(t), X_{2}(t)\right)$. It follows that an infinite number of these events will occur, $P$-a.s. The same is true when the roles of $X_{1}$ and $X_{2}$ are reversed. Thus (3.25) holds.

(v) According to (3.25), used repeatedly, there are points $\left(x_{j, k}, t_{k}\right)$ on the paths of $\dot{X}_{j}$ such that $x_{1, k}^{d}=x_{2, k}^{d} \rightarrow \infty$. Using Lemma 3.21, as in the argument of section (iii) above, we get that $\left(x_{1, k}, t_{k}\right)$ and $\left(x_{2, k}, t_{k}\right)$ have the same limit in $\partial_{0}^{M} \dot{D}_{f}$. Thus, the limits of $\dot{X}_{1}(t)$ and $\dot{X}_{2}(t)$ in $\partial_{0}^{M} \dot{D}_{f}$, as $t \rightarrow \infty$, are the same. Since $\dot{X}_{1}$ and $\dot{X}_{2}$ are independent, the measure $\mu$ such that $h(x)=\int_{\partial_{0}^{M} \dot{D}_{f}} \dot{K}((x, 0), z) \mu(d z)$ must actually be supported on a singleton. That is, $h$ must be minimal as a parabolic function.

It is the use of Lemma 3.21 that requires the assumption that $f(u) \rightarrow 0$. If only $\lim \sup _{u \rightarrow \infty} f(u)<\infty$, we modify the argument as follows. For any $\varepsilon>0$,

$$
P_{h}^{x, t}\left(X\left(f\left(\left(x^{d}\right)^{2}\right)\right) \in B\left(\left(0, \ldots, 0, x+f\left(x^{d}\right)\right), \varepsilon f\left(x^{d}\right)\right)\right) \geq c(\varepsilon)>0,
$$

for every $(x, t) \in \dot{D}_{f}$. Let

$$
W_{\varepsilon} \stackrel{\mathrm{df}}{=} \inf \left\{t>0: X_{1}^{d}(t), X_{2}^{d}(t) \in B((0, \ldots, 0, u+f(u)), \varepsilon f(u)) \text { for some } u\right\} .
$$

Applying (3.30) to $x=X_{j}(W)$ and using another iterative argument, one can show that $P\left(W_{\varepsilon}<\infty\right)=1$ for every $\varepsilon>0$. Taking a sequence $\varepsilon_{k} \rightarrow 0$, now gives sequences $\left(x_{j, k}, t_{k}\right)$ on the paths of $\dot{X}_{j}$, such that

$$
x_{j, k} \in B\left(\left(0, \ldots, 0, u_{k}+f\left(u_{k}\right), \varepsilon_{k} f\left(u_{k}\right)\right),\right.
$$

where $u_{k} \rightarrow \infty$. An argument as in the proof of Lemmas (3.14) and 3.21 now shows that the $\left(x_{1, k}, t_{k}\right)$ and $\left(x_{2, k}, t_{k}\right)$ have the same limit in $\partial_{0}^{M} \dot{D}_{f}$. As before, this shows that $h$ is parabolically minimal.

\section{REFERENCES}

[An] A. Ancona, Principe de Harnack à la frontière et théorème de Fatou pour un opérateur elliptique dans un domaine lipschitzien, Ann. Inst. Fourier 28 (1978), 169-213. MR 82a:31017; MR 80d:31006

[Ar] D.G. Aronson, Non-negative solutions of linear parabolic equations, Ann. Scuola Norm. Sup. Pisa 22 (1968), 607-694. MR 55:8554; MR 55:8553

[BBB] R. Bañuelos, R. Bass and K. Burdzy, Hölder domains and the boundary Harnack principle, Duke Math. J. 64 (1991), 195-200. MR 92g:35077

[BD] R. Bañuelos and B. Davis, A geometrical characterization of intrinsic ultracontractivity for planar domains with boundaries given by the graphs of functions, Indiana U. Math. Jour. 41 (1992), 885-912. MR 94g:60142

[BB1] R. Bass and K. Burdzy, A boundary Harnack principle in twisted Hölder domains, Ann. Math. 134 (1991), 253-276. MR 92m:31006

[BB2] R. Bass and K. Burdzy, Lifetimes of conditioned diffusions, Probab. Theory Related Fields 91 (1992), 405-443. MR 93e:60155

[BTW] K. Burdzy, E. Toby and R.J. Williams, On Brownian excursions in Lipschitz domains. Part II. Local asymptotic distributions, Seminar on Stochastic Processes 1988 (E. Cinlar, K.L. Chung, R. Getoor, J. Glover, eds.), Birkhäuser, Boston, 1989, pp. 55-85. MR 90k:60142

[C] M. Cranston, Lifetime of conditioned Brownian motion in Lipschitz domains, Z. Wahrschein. Verw. Gebiete 70 (1985), 335-340. MR 87a:60088

[CM] M. Cranston and T.R. McConnell, The lifetime of conditioned Brownian motion., Z. Wahrschein. Verw. Gebiete 65 (1983), 1-11. MR 85d:60150

[Dg] B. Dahlberg, Estimates of harmonic measure, Arch. Rat. Mech. Anal. 65 (1977), 275-288. MR 57:6470 
[Dv] B. Davis, Conditioned Brownian motion in planar domains, Duke Math. J. 57 (1988), 397-421. MR 89j:60112

[DZ] B. Davis and B. Zhang, Moments of the lifetime of conditioned Brownian motion in cones, Proc. Amer. Math. Soc. 121 (1994), 925-929. MR 94i:60097

[Db] J.L. Doob, Classical Potential Theory and Its Probabilistic Counterpart, Springer, New York, 1984. MR 85k:31001

[FGS] E.B. Fabes, N.Garofalo and S. Salsa, A backward Harnack inequality and Fatou theorem for nonnegative solutions of parabolic equations, Illinois J. Math. 30 (1986), 536-565. MR 88d:35089

[FO] B. Fristedt and S. Orey, The tail $\sigma$-field of one-dimensional diffusions, Stochastic Analysis (A. Friedman and M. Pinsky, eds.), Academic Press, New York, 1978, pp. 127-138. MR 80d:60098

[KL] U. Küchler and U. Lunze, On the tail $\sigma$-field and minimal parabolic functions for onedimensional quasi-diffusions, Z. Wahrschein. Verw. Gebiete 51 (1980), 303-322. MR 82f: 60165

[MSW] P.A. Meyer, R.T. Smythe and J.B. Walsh, Birth and death of Markov processes, Proc. 6th Berkeley Symp. Math. Stat. Prob., vol. III, Univ. of California Press, Berkeley, CA, 1972, pp. 295-305. MR 53:9392

[P] R. Pinsky, Positive Harmonic Functions and Diffusion, Cambridge Univ. Press, Cambridge, 1995. MR 96m:60179

[Rg] L.C.G. Rogers, Coupling and the tail $\sigma$-field of a one-dimensional diffusion, Stochastic calculus in application (J.R. Norris, ed.), Pitman Res. Notes Math., vol. 197, Longman Sci. Tech., Harlow, England, 1988, pp. 78-88. MR 90f:60142

[Rs] U. Rösler, The tail $\sigma$-field of a time-homogeneous one-dimensional diffusion processes., Ann. Prob. 7 (1979), 847-857. MR 81e:60086

[W] J.-M. G. Wu, Comparison of kernel functions, boundary Harnack principle, and relative Fatou theorem on Lipschitz domains, Ann. Inst. Fourier Grenoble 28 (1978), 147-167. MR 80g:31005

[X] J. Xu, The lifetime of conditioned Brownian motion in domains of infinite area, Prob. Theory Related Fields 87 (1991), 469-487. MR 92d:60086

[Z] B. Zhang, On the variances of occupation times of conditioned Brownian motion, Trans. Amer. Math. Soc. 348 (1996), 173-185. MR 96e:60151

Department of Mathematics, Box 354350, University of Washington, Seattle, WashINGTON 98195-4350

E-mail address: burdzy@math.washington.edu

Department of Mathematics and Statistics, York University, Toronto, Ontario, CANADA M3J 1P3

E-mail address: salt@nexus.yorku.ca 